\title{
Die Reform des deutschen Steuerrechts
}

\author{
von Paul Kirchhof
}

\begin{abstract}
Das Steuerrecht wird immer komplizierter, unverständlicher und willkürlicher. Ständige Gesetzesänderungen machen es zu einem Recht auf Rädern, das langfristige Planungen gefährdet, unternehmerische Kraft sinnlos bindet und die Vertrauenswürdigkeit des Staates an einer sensiblen Stelle des rechtlichen Alltags gefährdet. Deshalb ist es Aufgabe der Wissenschaft, ein einfaches, systematisches und langfristig verbindliches Steuergesetzbuch zu entwerfen, das den steuerlichen Belastungsgrund einsichtig macht und dem Wirtschaftsleben eine verlässliche Rechtsgrundlage bietet. Die vorgeschlagene Reform setzt auf eine vereinfachte Besteuerung des Einkommens, der im Umsatz ersichtlichen Kaufkraft, der Erbschaft und Schenkung sowie des Verbrauchs von Energie und Genussmitteln.
\end{abstract}

German tax law is getting increasingly complicated, unintelligible, and arbitrary. Continuing legislative changes transform it into a moving target that interferes with long-term planning, demands an unnecessary degree of attention from businesses, and endangers the trustworthiness of the state in a sensitive area of the legal system. Thus, it is the task of academic research to develop a simple and systematic unified code of federal tax law (Bundessteuergesetzbuch, BStGB) that aims at long term stability, seeks to make intelligible the cause for taxation and offers a reliable legal foundation for businesses. The proposed reform draws on a simplified taxation of income, purchasing power, inheritances and gifts, and the consumption of specific goods, such as energy and luxury articles.

\section{Einleitung}

Das gegenwärtige Steuerrecht wird allgemein als unzulänglich empfunden, weil es unübersichtlich ist, die Freiheit des Wirtschaftens erschwert und hemmt, in seinem ständigen Wechsel langfristige Planungen kaum zulässt, mit seinen Lenkungstatbeständen Freiheit beengt und nicht selten in die ökonomische Torheit führt. Dennoch erwächst aus diesen Rechtsmängeln bisher kein kraftvoller politischer Reformimpuls, weil die Parteien- und Verbändedemokratie auf Privilegienund Sondertatbeständen beharrt, eine folgerichtige Besteuerung nach finanzieller Leistungsfähigkeit den Bedrängnissen eines weltoffenen Marktes ausgesetzt ist und eine Steuer mit maßvollen Steuersätzen an den ständig wachsenden Leistungserwartungen gegenüber dem Staat zu scheitern droht. 
Hier liegt die Aufgabe der Wissenschaft. Sie muss der Öffentlichkeit und der Politik nachweisen, dass ein maßvolles und gleichmäßiges Steuerrecht möglich ist, das Gesetz einen verständlichen und einsichtigen Belastungsgrund für jede Steuer entwickeln kann, ein einfaches und folgerichtiges Steuerrecht Freiheit, Rechtssicherheit und Planbarkeit mehrt, schließlich das Ideal der Allgemeinheit und Privilegienfeindlichkeit des Gesetzes im Steuerrecht unerlässlich ist.

\section{Die Steuer als Preis der Freiheit}

\section{Staatsunternehmen oder Steuern}

Der Verfassungsstaat garantiert die Berufs- und Eigentümerfreiheit, belässt deshalb die Produktionsfaktoren Arbeit und Kapital in privater Hand und verzichtet strukturell auf staatliche Unternehmen, die dem Staat Erträge bringen könnten. Die Arbeitskraft und die Wirtschaftsgüter werden grundsätzlich von den freiheitsberechtigten Menschen privat genutzt; der freiheitsverpflichtete Staat ist insoweit von der Bewirtschaftung dieser Ertragsquellen ausgeschlossen. Er ist darauf verwiesen, durch Steuern am Erfolg privaten Wirtschaftens teilzuhaben. Der Steuerertrag hängt vom Ergebnis privatwirtschaftlicher Leistungen ab; öffentliche und private Hand sind in gemeinsamem Interesse an einer prosperierenden Wirtschaft verbunden.

Wenn die Gesellschaft grundrechtliche Freiheit beansprucht, insbesondere die wirtschaftlichen Freiheiten der Berufs-, Eigentümer- und Vereinigungsfreiheit wahrnimmt und in dieser wirtschaftlichen Freiheit zugleich eine Grundlage sittlicher Freiheit sieht, ${ }^{1}$ ist dem Staat ein allein in seiner Hoheitsgewalt begründeter Zugriff auf seine Bürger und deren Eigentum verwehrt. Der Verfassungsstaat darf sich nur in Achtung vor der Freiheit und Gleichheit seiner Bürger finanzieren, deshalb nur steuerlich an dem von den Bürgern in Freiheit erwirtschafteten und freiheitlich geschützten Eigentum teilhaben. Dabei greift der freiheitliche Staat in bemerkenswerter Liberalität auf den wirtschaftlichen Erfolg der Freiheitsberechtigten zu, nicht auf ihre persönliche Erwerbskraft oder auf ein Äquivalent für empfangene Staatsleistungen. Wer in Deutschland mit staatlicher Unterstützung studiert und deshalb glänzende Erwerbsmöglichkeiten erworben hat, sich dann aber für eine zweijährige Weltreise entscheidet und dementsprechend die Steuerzahlung im Inland verweigert, wird in dieser seiner Freiheit respektiert.

1 Schön, W.: Steuergesetzgebung zwischen Markt und Grundgesetz, in: Mellinghoff, R./Morgentaler, G./Puhl, T. (Hg.): Die Erneuerung des Verfassungsstaates, 2003, 143 (148). 
Eine Kopfsteuer, die in den USA lange als Bedingung des Wahlrechts galt (poll tax), in Großbritannien noch Ende der 1980er-Jahre als Gemeindekopfsteuer neu eingeführt wurde und heute noch in einigen Schweizer Kantonen gilt, ${ }^{2}$ erscheint dem modernen Verfassungsstaat unvertretbar, weil sein Freiheitsverständnis keine allgemeine Pflicht anerkennt, sich für den Staat anstrengen und erwerben zu müssen. Außerdem können viele Menschen wegen ihres Alters, wegen unzulänglicher Ausbildung, Krankheit oder einem Mangel an Möglichkeiten zur Arbeit nicht am Erwerbsleben teilnehmen und beanspruchen deswegen die Hilfe des sozialen Staates. Die Rechtsgemeinschaft bietet dem einzelnen generell Erwerbs- und Tauschmöglichkeiten, die er nutzt. In dieser Nutzungsmöglichkeit ist er der Gemeinschaft verpflichtet. ${ }^{3}$

\section{Teilhabe am Erfolg privaten Wirtschaftens}

Die Steuer soll dem Staat nicht in beliebiger Weise - durch Raub, Brandschatzung oder Konfiskation - Erträge zuführen, sondern den freiheitlichen Verfassungsstaat am Erfolg privaten Wirtschaftens teilhaben lassen. Besteuerungsziel ist nicht ein beliebiges Aufkommen, sondern ein staatlicher Anteil am Erfolg individuellen Arbeitens, individueller Eigentumsnutzung und individueller Kaufkraft. Die Steuer rechtfertigt sich nicht, weil sie staatliche Erträge erzielt, sondern weil sie den Staat freiheitskonform am Ergebnis individuellen Wirtschaftens teilhaben lässt. Diese Rechtfertigung lässt sich im Steuergegenstand vertiefen: Der Mensch erzielt Einkommen dank eigener Leistung, stützt sich dabei aber auf einen Markt von Anbietern und Nachfragern, auf eine Rechtsordnung zur Begründung und Durchsetzung vertraglicher Verbindlichkeiten, auf ein Banken- und Währungswesen, auf durch Schulen und Hochschulen gut ausgebildete Arbeitnehmer, auf einen gewerblichen Rechtsschutz, auf eine friedenstiftende Rechtsordnung, auf Rechtssicherheit in der Nationalökonomie und dem durch sie eröffneten Weltmarkt. Allein eine wertvolle Leistung - das zu seinen Lebzeiten noch nicht verstandene Wort des Dichters, die ihrer Zeit vorauseilende Melodie eines Komponisten, das dem Stilempfinden erst der Zukunft entsprechende Gemälde eines Malers - führt noch nicht zu einem Einkommen. Einkommen entsteht erst, wenn die Rechtsgemeinschaft eine Leistung durch Entgelt aner-

2 Tipke, K.: Die Steuerrechtsordnung, Band I, 2000, 2. Aufl., 473 ff.

3 Vgl. Vogel, K.: Rechtfertigung der Steuer: Eine vergessene Vorfrage, in: Der Staat 1986, 481 (496 f.); Kirchhof, P.: Empfiehlt es sich, das Einkommensteuerrecht zur Beseitigung von Ungleichbehandlungen und zur Vereinfachung neu zu ordnen?, Gutachten F für den 57. Deutschen Juristentag 1988, F 16 f. 
kennt. Ebenso begründet allein die Kaufkraft - wenn sie in der Wüste eingesetzt werden sollte - noch keinen Umsatz; erst die Infrastruktur eines umfassenden und allgemeinen Leistungsangebotes des Marktes erlaubt es dem Nachfrager, seine Kaufkraft in Gegenleistungen einzutauschen. Deshalb rechtfertigt sich die „,voraussetzungslose“ Steuer ${ }^{4}$ als Teilhabe am jeweiligen individuellen Erfolg privatnützigen Wirtschaftens, der sich seinerseits auf die von der Rechtsgemeinschaft bereitgestellte Freiheitsstruktur von Marktordnung, Frieden, Rechtssystem, Schul- und Ausbildungswesen stützt.

Die je nach individueller Finanzkraft bemessene Einkommensteuer erfasst die Steuerpflichtigen in ihrer freiheitlich hergestellten Verschiedenheit, die Umsatzsteuer die Nachfrager in ihrer Kaufkraft, einem anonymen Konsum. Die Struktur der Einkommensteuer, die den existenznotwendigen Bedarf verschont und Einkommen progressiv besteuert, entlastet die Finanzschwachen. Eine Umsatzsteuer, die Börsen- und Finanzumsätze ausnimmt, benachteiligt die Finanzschwachen. Die Finanzstarken hingegen werden bei den indirekten Steuern bevorzugt, bei den direkten Steuern zwar progressiv, oft aber in einer verkürzten Bemessungsgrundlage belastet. Der urdemokratische Gedanke, das Wahlrecht entspreche der Steuerpflicht, ${ }^{5}$ und der freiheitsgerechte Gedanke, alle Staatsbürger seien je nach ihrer unterschiedlichen wirtschaftlichen Lage für die Finanzierung der Gemeinlasten gleich verantwortlich, ${ }^{6}$ haben im geltenden Steuerrecht noch nicht zu einem Zusammenklang gefunden. Dieser Strukturfehler betrifft die gerechte Verteilung der Lasten, nicht die aus Steuererträgen erzielte Handlungsmacht des Staates. Die ,,voraussetzungslose“ Steuer sichert die finanzielle Handlungsfähigkeit der parlamentarischen Demokratie. Die grundsätzliche Zweckfreiheit der Steuer $^{7}$ stützt die Budgethoheit des Parlaments und die innere Unbefangenheit des Staates gegenüber seinen Finanziers.

4 Mayer, O.: Deutsches Verwaltungsrecht, 1924, Band. 1, 316; Wendt, R.: Finanzhoheit und Finanzausgleich, in: HStR IV, 1999, 2. Aufl., § 104, Rn. 16 ff.; Waldhoff, C.: Grundzüge des Finanzrechts des Grundgesetzes, in: HStR V, 2007, 3. Aufl., § 116 Rn. 85.

5 „No taxation without representation”, vgl. dazu Tipke, K, a.a.O., 474.

6 Birk, D.: Das Leistungsfähigkeitsprinzip als“ Maßstab der Steuernormen, 1983; Walz, W.R.: Steuergerechtigkeit und Rechtsanwendung, 1980; Lehner, M.: Einkommensteuerrecht und Sozialhilferecht, 1993; Tipke, K., a.a.O., 479 f.

7 Waldhoff, C.: Verfassungsrechtliche Grenzen der Zwecksteuer, in: StuW 2002, 285. 


\section{Die unausweichliche Steuerlast}

Die Rechtfertigung der Steuer im Belastungsgrund des Steuergegenstandes fordert die unausweichliche Steuerlast. ${ }^{8}$ Der Steuerzahler wird gleichheitsgerecht belastet, weil er sich im Einkommen, in der Erbmasse, im Umsatz und in einem Sonderbedarf als belastbar erweist, nicht weil er sich willentlich für eine Besteuerung angeboten hat. Dieses Postulat der Unausweichlichkeit der Last ist die verfassungsrechtliche Antwort auf die größte Schwäche des gegenwärtigen Besteuerungswesens, die Vermeidbarkeit der Steuer durch steuerbewusste Sachverhaltsgestaltung. Der Steuerpflichtige hat zu zahlen, weil er finanzwirtschaftlich leistungsfähig ist, nicht weil er gesetzlich angebotene oder zugelassene Ausweichstrategien nicht hinreichend genutzt hat. Die Steuer wird vom Staat gleichund freiheitsgerecht zugeteilt, nicht ähnlich dem Schachspiel zwischen Finanzamt und Steuerpflichtigen mit Witz, Geschick und Finten ausgespielt. Den Steuerpflichtigen erreicht die Last, weil er wirtschaftlichen Erfolg hatte, nicht weil er sich intellektuell vorwerfen müsste, er hätte seinen Erfolg steuertaktisch nur ungenügend bemäntelt.

Der Gesetzgeber muss die Steuerpflicht deshalb so ausgestalten, dass jeder Pflichtige im tatsächlichen Belastungserfolg gleich zur Finanzierung der Staatsaufgaben herangezogen wird. ${ }^{9}$ Das materielle Steuergesetz hat im Tatbestand den Belastungsgrund zu nennen, der die grundrechtserhebliche Verschiedenheit nach Einkommen, Vermögen oder Nachfragekraft $-{ }^{10}$ erfasst, in dieser Tatbestandlichkeit durch Steuergestaltung nicht verändert werden kann, der Tatbestand außerdem die Gewähr seiner regelmäßigen Durchsetzbarkeit soweit wie möglich in sich trägt. ${ }^{11}$

Deshalb hat der Steuergesetzgeber vor allem deutlich zu machen, wann er insbesondere bei statusbegründenden Rechtsakten - bewusst an zivilrechtliche Vorgaben anknüpft, und wann er - wie in der Regel - der Besteuerung eine eigenständige steuerjuristische Betrachtungsweise zugrunde legt. ${ }^{12}$ Der Gesetzgeber kann den Belastungstatbestand auch durch Typisierung unausweichlich voll-

8 BVerfGE 96, 1 (6) - Arbeitnehmerfreibetrag.

9 BVerfGE 84, 239 (268) - Zinsbesteuerung; 93, 121 (134) - Vermögensteuer.

10 BVerfGE 93, 121 (134) - Vermögensteuer.

11 BVerfGE 84, 239 (272) - Zinsbesteuerung.

12 BVerfG, Beschluss vom 27.12.1991 - 2 BvL 72/90, NJW 1992, 1219 f. - Grunderwerbsteuer. 
ziehbar machen. ${ }^{13}$ Schließlich ist die Erhebungsform der Quellensteuer ein geeignetes Instrument, um die Unausweichlichkeit im Gesetzesvollzug zu gewährleisten. $^{14}$

Das Steueraufkommen dient der fortlaufenden, gegenwartsnahen Finanzierung staatlicher Aufgaben. Der Gleichheitssatz fordert insoweit auch eine Belastungsgleichheit in der Zeit. Die Steuerpflichtigen sind je nach ihrer finanziellen Leistungsfähigkeit nicht nur in ihrem Lebenseinkommen, ${ }^{15}$ sondern je nach gegenwärtigen Markteinnahmen, Existenzbedürfnissen und Erwerbsaufwendungen gleichmäßig zu belasten. ${ }^{16}$ Insbesondere der Einkommensteuerpflichtige soll mit seinem gegenwärtigen Einkommen zur Deckung des gegenwärtigen staatlichen Finanzbedarfs beitragen. Dementsprechend ist die Einkommensteuer in ihrer Ausgestaltung als Jahressteuer und in ihrer kontinuierlichen Erhebung auch auf eine Belastungsgleichheit in der Zeit angelegt. ${ }^{17}$ Sie dient als periodisch wiederkehrende Belastung gegenwärtiger Einkommen der Ausstattung des Staates mit Finanzmitteln für das jeweilige Haushaltsjahr. ${ }^{18}$

Die wirksamste Form eines gegenwartsnahen Gesetzesvollzugs bietet die Quellensteuer, die das Einkommensteuergesetz für die Lohnsteuer und die Einkünfte aus Kapitalvermögen kennt. Ist der Quellenabzug bei anderen Einkünften nicht möglich oder nicht gesetzlich vorgesehen, werden Steuerzahlungen nicht in gleicher Gegenwartsnähe erbracht. Das gesetzliche Ziel einer gegenwartsnahen und unausweichlichen Steuererhebung ${ }^{19}$ wird nicht vollständig verwirklicht. Dies sollte dem Gesetzgeber Anlass sein, die Zeitgleichheit und Unausweichlichkeit der Steuerlast zu verbessern.

Das Bewertungsrecht muss das Bewertungsgut realitätsgerecht abbilden und die Entwicklung im späteren Zeitverlauf aufnehmen. Ein Auto hat bei der Anschaffung den Wert des Kaufpreises, veranlasst später bei der Entsorgung aber noch Kosten. Sand in der Wüste ist Gemeingut, wird im Betonmischer zum Wirtschaftsgut, Sand im Getriebe ist ein Ärgernis. Das Steuerrecht muss diese Unter-

13 Vgl. BVerfGE 82, 159 (185 f.) - Absatzfonds; 87, 153 (172) - Grundfreibetrag; 96, 1 (6) - Arbeitnehmerfreibetrag; 101, 297 (309) - Arbeitszimmer.

14 BVerfGE 96, 1 (7 f.) - Arbeitnehmerfreibetrag, für die Lohnsteuer; 84, 239 (282 f.) - Zinsurteil, für die Einkünfte aus Kapitalvermögen.

15 So aber Tipke, K., a.a.O., 754 ff. mit weiteren Nachweisen.

16 BVerfGE 87, 153 (169) - Grundfreibetrag.

17 BVerfGE 96, 1 (7) - Arbeitnehmerfreibetrag.

18 BVerfGE 87, 153 (179) - Grundfreibetrag.

19 BVerfGE 96, 1 (8) - Arbeitnehmerfreibetrag. 
schiede in abstrakten Maßstäben wirklichkeitsnah erfassen, sodann die Entwicklung der Werte - etwa eines Grundstücks - in der Zeit mitschreitend nachzeichnen. $^{20}$

Das Verfahrensrecht ist so zu regeln, dass das materielle Gesetz auch tatsächlich verlässlich vollzogen werden kann; strukturelle Erhebungsmängel führen zur Unvereinbarkeit des Gesetzes mit dem Gleichheitssatz und begründen eine gesetzgeberische Pflicht zur Normensanierung. ${ }^{21}$

\section{Das einfache und verständliche Steuerrecht}

Die steuerliche Gesetzgebungspraxis der Gegenwart gibt Anlass, das rechtsstaatliche Erfordernis des einfachen und verständlichen Gesetzes nachdrücklich in Erinnerung zu rufen. Ein unverständliches Gesetz ist nicht ordnungsgemäß beschlossen (Art. 77 Abs. 1 Satz 1 GG) und nicht ordnungsgemäß zustande gekommen (Art. 78 GG), weil weder die Abgeordneten des Bundestages noch die Mitglieder des Bundesrates ihren Entscheidungsgegenstand verstehen konnten. Es ist nicht ordnungsgemäß verkündet (Art. 82 Abs. 1 Satz 1 GG), weil im Bundesgesetzblatt zwar ein Text, nicht aber ein die Steuerlast rechtfertigender Belastungsgedanke veröffentlicht worden ist. Die Regelung bietet auch keinen hinreichenden Maßstab für die Verwaltung der Einkommensteuer durch die Landesfinanzbehörden (Art. 108 Abs. 2 Satz 1 GG), wenn das Bundesfinanzministerium die gesetzliche Vorschrift nicht in deutscher Sprache, sondern nur durch Rechenhilfen erläutern kann. Die Steuer steht unter Gesetzes-, nicht unter Computervorbehalt. Vor allem aber verlangt das aus Art. 20 Abs. 3 GG folgende rechtsstaatliche Bestimmtheitsgebot, dass der Betroffene aus dem Gesetz die Rechtslage erkennen und nach ihr sein Verhalten ausrichten kann. Dies gilt im Grundsatz der Tatbestandsmäßigkeit der Besteuerung insbesondere für das Steuerrecht, weil der Inhalt einer Steuerschuld weitgehend von der gesetzlichen Entscheidung abhängt, zudem die steuerlichen Erklärungspflichten strafbewehrt sind, der Steuerpflichtige also im Vorhinein nach Art. 103 Abs. 2 GG die an ihn gestellten Anforderungen erkennen können muss. ${ }^{22}$

20 BVerfGE 93, 121 (136 f.) - Vermögensteuer; 117, 1-70 - Bewertung im Erbschaftsteuerrecht.

21 BVerfGE 84, 239 (272 ff.) - Zinsbesteuerung; anders die unzulängliche Vollziehbarkeit wegen fehlender Rechtstreue des Steuerschuldners, BVerfG, Urteil vom 12.4.1996 - 2 BvL 18/93, NJW 1996, 2086 f. - Bordellbetrieb.

22 Vgl. BVerfGE 99, 216 (243) $\square$ Kinderbetreuungskosten. 
Im übrigen droht der Rechtsstaat zu scheitern, wenn der Bürger an einer zentralen Bewährungsprobe des Rechts, dem steuerlichen Eingriff, den rechtfertigenden Grund für die Belastung nicht erkennen kann, die Belastungsunterschiede ihm nicht einsichtig sind, das Belastungsmaß im Steuersatz (vgl. § 32 a EStG) für ihn unverständlich bleibt. Der Gesetzgeber verweigert insoweit den Dialog mit dem Steuerpflichtigen. Der Steuerpflichtige muss den Eindruck gewinnen, er solle sich dem Gesetz unterwerfen, ohne es verstehen zu können.

\section{Das Reformanliegen}

Der Heidelberger Entwurf eines Bundessteuergesetzbuchs (BStGB) setzt sich zum Ziel, das geltende Steuerrecht in einem Entwurf eines einsichtigen Steuergesetzbuchs zu vereinfachen. Dabei werden die erprobten und bewährten Prinzipien des deutschen Steuerrechts gewahrt, Verfremdungen, Lenkungen und Bevorzugungen jedoch beendet. Das Steuerrecht ist geeignet, in einer Gesamtkodifikation geordnet, systematisch erschlossen, sprachlich erneuert zu werden.

Der historisch gewachsene Besteuerungsgrund muss wieder erkennbar werden, so dass jeder Steuerpflichtige nachvollziehen kann, welche Last er zu tragen hat und was der rechtfertigende Grund für den staatlichen Zugriff ist. So wird die Besteuerung für alle möglichst unausweichlich und gerecht. Sachverhaltsgestaltungen, die allein aus steuerlichen Gründen gewählt werden, verlieren in einem reformierten Steuerrecht ihr Gewicht. Die Gleichheit der Last wird gesichert, je weniger die Höhe der Steuern durch das rechtliche Gewand eines wirtschaftlichen Vorgangs beeinflusst werden kann. Wird die Steuer auf alle Schultern verteilt, wird sie auch maßvoll sein.

Die Kodifikation eines Bundessteuergesetzbuches fasst die derzeit mehr als 200 Steuergesetze zu einem einzigen Steuergesetzbuch zusammen. Zusätzlich reduziert es die mehr als 30 Bundessteuern auf vier; eine Einkommensteuer, eine Umsatzsteuer, eine Erbschaftsteuer (einschließlich Schenkungsteuer) sowie eine Sonderverbrauchsteuer, in der einige der gegenwärtigen Verbrauch- und Verkehrsteuern systematisch erfasst werden. Eine Gemeindeertragsteuer ersetzt die Gewerbesteuer durch ein kommunales Hebesatzrecht. Den vier materiellen Steuergesetzen wird ein Allgemeiner Teil vorangestellt, der Grundsätze für alle Steuern regelt.

Die Besteuerung von Einkommen, Erbschaft und Kaufkraft wirkt freiheitsschonend, weil sie den Steuerpflichtigen immer dann belastet, wenn sich seine Leis- 
tungsfähigkeit zu seinen Gunsten verbessert hat. Auf Substanzsteuern hingegen wird grundsätzlich verzichtet. Diese Steuern stellen lediglich fest, dass jemand über Vermögen, Gewerbekapital oder Grundbesitz verfügt und deshalb zum Objekt staatlicher Besteuerung werden soll. ${ }^{23}$ Die Garantie der Berufs-, Eigentümer- und Vereinigungsfreiheit gibt das Recht, sich von anderen zu unterscheiden. Unterschiede im Einkommen, im Erbanfall, im Umsatz sind freiheitlich gerechtfertigt, deswegen noch nicht Grund für staatliche Umverteilung. Selbstverständlich soll die staatliche Ausgabenpolitik die in Einkommen und Vermögen angelegten Entfaltungschancen angleichen. Doch ein Steuerzugriff allein deshalb, weil der Pflichtige reicher geworden ist, würde sich allenfalls rechtfertigen, wenn die Gründe der Bereicherung freiheitsgerecht ermittelt würden. Doch bisher ist nicht tatbestandserheblich, ob das Einkommen unter großer Anstrengung oder leichter Hand erworben ist, ob die Erbschaft durch Pflege des Erblassers oder durch Leistungen in seinem Betrieb verdient, lediglich als Sympathiezuwendung zu verstehen oder kraft Gesetzes angerechnet worden ist, ob die bei der Umsatzsteuer erfasste Kaufkraft durch Arbeit, Darlehen oder Almosen gewonnen und zu welchen Zwecken - zur Deckung eines Elementarbedarfs oder zum Erwerb von Luxusgütern - sie eingesetzt wird.

Ein vereinfachtes Steuersystem gibt der Wirtschaft einen Prosperitätsimpuls. Der erwerbswirtschaftlich tätige Mensch hat den Kopf wieder frei für seinen Markt, sein Produkt und seine Kunden. Ein niedriger Steuersatz stärkt den Standort Deutschland in der Weltwirtschaft und wirkt attraktiv für qualifizierte Arbeitskräfte und Kapital.

\section{Allgemeiner Teil des Bundessteuergesetzbuchs}

Der Allgemeine Teil des Bundessteuergesetzbuches enthält die für alle Steuern geltenden Regelungen des Bundessteuergesetzbuches.

\section{Obergrenze der Gesamtbelastung, Steuerkonkurrenzlehre}

Die Steuer überführt privates Eigentum in die öffentliche Hand, muss bei diesen Belastungen den Maßstäben der Grundrechte genügen. Der Eigentumsschutz des Art. 14 GG sichert dem Eigentümer einen Freiraum finanzwirtschaftlichen Han-

23 So aber die Reformanregung der OECD, die beobachtet, dass in Deutschland die Steuererträge aus Substanzsteuern gering seien, OECD: Economic Surveys - Germany, April 2008, 59 ff. 
delns, schützt damit auch das Geldeigentum. ${ }^{24}$ Der Eigentumsschutz gegenüber der Besteuerungsgewalt mäßigt den Steuerzugriff, unterbindet ihn nicht. Die Steuer ist der Preis der Eigentümerfreiheit. Wenn die Garantie des privatnützigen Eigentums den Produktionsfaktor Eigentum strukturell in privater Hand belässt, den Staat also nicht durch Staatsunternehmen, sondern durch Teilhabe am Erfolg privaten Wirtschaftens finanziert, fordert die Eigentumsgarantie die Steuer. Der freiheitliche Staat ist Steuerstaat.

Allerdings muss sich die Freiheitlichkeit der Steuer in ihrem Maß bewähren. Das Bundesverfassungsgericht hat von Anfang an betont, dass die Steuer nicht ,,konfiskatorisch“, ,erdrosselnd“ sein dürfe. ${ }^{25}$ Später hat es die Verhältnismäßigkeit des Steuereingriffs ,,in der Nähe einer hälftigen Teilung zwischen privater und öffentlicher Hand“ verdeutlicht, ${ }^{26}$ jüngst aber wieder auf das allgemeine Verhältnismäßigkeitsprinzip zurückgenommen. ${ }^{27}$ Anerkannt ist jedenfalls, dass die Steuer die Sozialpflichtigkeit (Art. 14 Abs. 2 GG) realisiert und nicht in die Intensität einer Enteignung (Art. 14 Abs. 3 GG) übergreifen darf; diese wäre entschädigungspflichtig, machte die Steuer damit sinnlos.

Der Gesetzgeber hat als Erstinterpret der Verfassung die Obergrenze der Gesamtbelastung zu definieren. Eine das Steuerrecht dirigierende Grundsatznorm bindet - für die Dauer ihrer Geltung - den Gesetzgeber selbst. ${ }^{28}$ Das BStGB setzt der steuerlichen Gesamtbelastung in der Hälfte des Jahreseinkommens eine Grenze und verweist damit auf das Erfordernis, die Staatsaufgaben strukturell zu überprüfen. $^{29}$

Trotz einer generell maßvollen Besteuerung kann das Gesetz im Einzelfall eine Überlast veranlassen. Auch in diesem Fall ist die Grenze der Zumutbarkeit zu wahren; eine staatliche Maßnahme darf den Betroffenen nicht übermäßig belasten. ${ }^{30}$ Die durch die Besteuerung entstehende Härte wird unzumutbar, wenn

BVerfGE 93, 121 (137) - Vermögensteuer; 97, 350 (370) - Euro; 115, 97 (110) - Einkommen- und Gewerbesteuer.

BVerfGE 16, 147 (171) - Werkfernverkehr; 38, 61 (80 f.) - Leberpfennig.

BVerfGE 115, 97 (112) - Einkommen- und Gewerbesteuer.

Zur Bindung des Steuergesetzgebers an selbstgesetzte Prinzipien am Maßstab des Art. 3 Abs. 1 GG (Folgerichtigkeit) vgl. BVerfGE 84, 153 (179) - Zinsurteil; 98, 83 (100) - Landesrechtliche Abfallabgabe; 99, 280 (295) - Zulage Ost; 105, 73 (112) - Rentenbesteuerung; 105, 17 (47) Sozialpfandbriefe.

29 Zum Vorbehalt eines Zugriffs auf die Vermögenssubstanz in staatlichen Ausnahmelagen vgl. schon BVerfGE 93, 121 (138) - Vermögensteuer. 
der gesetzliche Belastungsgrund die individuell spürbare Last nicht rechtfertigt, die Auferlegung der Steuer also unangemessen ist. ${ }^{31}$ Die Verfassung gebietet in solchen Fällen, von der allgemeinen Norm des Steuerrechts abzuweichen und den Staatszugriff zu mäßigen. Die Grundsätze der Gesetzmäßigkeit der Verwaltung und der Gleichmäßigkeit der Besteuerung werden im BStGB im Dienst einer Individualgerechtigkeit gelockert. ${ }^{32}$ Liegt eine unzumutbare Härte vor, hat die Finanzbehörde den Anspruch aus dem Steuerschuldverhältnis zu stunden, zu mindern oder ganz zu erlassen.

Auch enthält der Allgemeine Teil erstmals Vorschriften, die das Verhältnis der einzelnen Steuern untereinander klären. So wird klargestellt, dass die Einkommensteuer und der Gemeindezuschlag nebeneinander erhoben werden. Beide Steuern haben dieselbe Bemessungsgrundlage, die Zuschlagsteuer stattet die Gemeinden mit einem eigenen Hebesatzrecht aus. Aufeinander abgestimmt werden auch die Einkommen- und die Erbschaft- und Schenkungsteuer. Beide Steuern besteuern den Vermögenszuwachs einer Person, folgen aber zwei eigenständigen Belastungsgründen: Die Einkommensteuer belastet das in der Rechtsgemeinschaft erworbene Eigentum, die Erbschaft- und Schenkungsteuer das von der Rechtsgemeinschaft geschützte angefallene Eigentum. Ihre Grundtatbestände sind so gefasst, dass sie sich gegenseitig ausschließen. Der Einkommensteuer unterliegen die Markteinkommen einer Person, der Erbschaft- und Schenkungsteuer unentgeltliche Zuwendungen. Einkünfte, die der Erblasser zu Lebzeiten erzielt hat, aber bis zu seinem Tod noch nicht versteuert hat, unterliegen beim Erben der Einkommensteuer. Diese Einkommensteuer ist dann von der erbschaftsteuerlichen Bereicherung abzuziehen. Problematisch ist auch der Fall, dass der Erbe nach dem Erwerb eine Erwerbsgrundlage, Anteile an einer steuerjuristischen Person oder ein Grundstück verkauft und darin enthaltene Erträge realisiert. Da die latente Ertragsteuer in der erbschaftsteuerlichen Bemessungsgrundlage enthalten ist, darf der Erwerber diese innerhalb eines Zwei-JahresZeitraums von seiner erbschaftsteuerlichen Bereicherung abziehen, um eine steuerliche Doppelbelastung zu vermeiden. Schließlich stellt das Gesetz klar, dass eine Sonderverbrauchsteuer einen Gegenstand zusätzlich zur Umsatzsteuer

31 Vgl. Grzeszick, B., in: Maunz, Th./Dürig, G. (Hg.): Grundgesetz-Kommentar, München, $2010,60$. Auflage, Art. 20, VII, Rn. 117.

32 Vgl. Rüsken, R., in: Klein, F.: Abgabenordnung, 2009, 10. Auflage, § 163 Rn. 6c; v. Groll, R., in: Hübschmann, W./Hepp, E./Spitaler, A.: Abgabenordnung/Finanzgerichtsordnung, 2010, § 227 AO, Rn. 31. 
belastet. Verbrauch- und Umsatzsteuern sollen nebeneinander erhoben werden, kumulativ belasten.

\section{Systematische Durchdringung der steuerlichen Rechtsquellen}

Das BStGB ordnet steuerlichen Rechts- und Erkenntnisquellen und bestimmt das Verhältnis des BStGB zum Europarecht und zum Völkerrecht. Für die innerstaatliche Rechtsetzung zeichnet sie die Kompetenzverteilung nach der Finanzverfassung nach und sucht bestehende Streitfragen gesetzlich zu regeln. Ein formalisiertes Verfahren regelt den Umgang mit Leitsatzentscheidungen des Bundesfinanzhofes sowie das Zusammenwirken von BFH und Finanzverwaltung.

\section{Gegenwartsnahe, verlässliche Besteuerung}

Das Steuerrecht teilt Verfügungsgewalt über Geld und damit ökonomische Handlungsfreiheit in der Zeit zu. ${ }^{33}$ Rechtfertigender Grund jeder Steuer ist die Belastbarkeit des Steuerpflichtigen in der Gegenwart. Finanziell leistungsfähig ist der Steuerpflichtige zu der Zeit, in der er Einkommen erzielt, Vermögensgüter als Erbschaft oder Schenkung erwirbt, individuelle Kaufkraft einsetzt. Je länger der Erwerbsvorgang zurückliegt, das Erworbene dem grundrechtlich geschützten Bestand individuell zugeordnet ist, umso mehr schwächt sich die Rechtfertigung der Steuer ab. Der Zeitablauf wirkt als rechtsverändernde Kraft, verlangt deswegen nach einer zeitlichen Begrenzung, bis zu der Ansprüche aus dem Steuerschuldverhältnis längstens geltend gemacht werden dürfen. Der Gedanke einer gegenwartsnahen Besteuerung bedingt kurze Verjährungsfristen, die nur ausnahmsweise verlängerbar sind.

Das BStGB regelt die Verjährung aller vermögensrechtlichen Ansprüche in einer einzigen Vorschrift, vermeidet gestreute Regelungen an verschiedenen Orten im Gesetz. Der Dualismus zwischen Festsetzungs- und Zahlungsverjährung wird aufgegeben. Die Vereinheitlichung zu einer Verjährungsfrist für einen Anspruch vereinfacht das Recht, verhindert ansonsten notwendige, doppelte Bestimmungen und stärkt die Rechtssicherheit. Die regelmäßige Verjährung dauert drei Jahre. Eine längere Frist - zwölf Jahre - gilt für hinterzogene Steuern. Gegenüber dem geltenden Recht reduziert sich die Anzahl der fristverlängernden Tatbestände. Ansprüche lassen sich nicht beliebig lange aufrechterhalten, ihre Ver- 
jährung nicht beliebig oft unterbrechen. Die Verjährung sichert die zeit- und gegenwartsnahe Besteuerung.

Die Verlässlichkeit der Rechtsordnung ist eine Grundbedingung freiheitlicher Verfassungen. ${ }^{34}$ Deshalb werden Verwaltungsakte bestandskräftig. Das BStGB stärkt die Bindungswirkung eines Bescheids und lässt Berichtigungen bestandskräftiger Bescheide nur unter engen Voraussetzungen zu. Eine Änderung des Verwaltungsakts zu Ungunsten des Steuerpflichtigen ist lediglich in Fällen zulässig, in denen der Finanzbehörde Tatsachen oder Beweismittel nachträglich bekannt werden, sich der steuerliche Sachverhalt rückwirkend geändert hat, die Finanzbehörde durch eine Außenprüfung neue Erkenntnisse gewonnen hat oder der Widerruf vorbehalten war. Zugunsten des Steuerpflichtigen kann ein Verwaltungsakt nur geändert werden, wenn dieser die Rechtswidrigkeit nicht bereits in dem dafür vorgesehenen Rechtsbehelfsverfahren hätte abwenden können.

Andererseits fordert das Selbstveranlagungsverfahren, das im BStGB für die Einkommensteuer, die Umsatzsteuer und die Sonderverbrauchsteuer vorgesehen ist, eine Prüfung dieser selbstbelastenden Erklärung. Ist der Steuerpflichtige verpflichtet, die Steuer selbst zu berechnen, anzumelden und zum Fälligkeitszeitpunkt zu entrichten, gilt für die Steueranmeldung ein Berichtigungsvorbehalt. Die Finanzbehörde kann bei der erstmaligen Überprüfung der Steueranmeldung die materiell richtige Entscheidung in Form eines berichtigenden Verwaltungsakts treffen.

\section{Gemeinnützigkeitsrecht}

Körperschaften, die gemeinnützige, mildtätige oder kirchliche Zwecke verfolgen, genießen weitgehende Steuererleichterungen. ${ }^{35}$ Wer an solche Organisationen spendet, kann derzeit die Spende von seiner steuerlichen Bemessungsgrundlage abziehen und so seine Steuerschuld mindern. ${ }^{36}$ Die steuerliche Spendenförderung verringert die Bemessungsgrundlage für die Einkommensbesteuerung um den Betrag der Spende und führt so beim Spender zu einer Steuerersparnis, deren Höhe davon abhängt, welchem Steuersatz der Spender unterliegt. Dieses steuerliche Anreizsystem ist wenig transparent und hat in einem progressiven Steuertarif den unerwünschten Nebeneffekt, dass jeder Euro, der von einem einkom- 
mensstarken Steuerpflichtigen gespendet wird, stärker steuerlich gefördert wird als bei einem einkommensschwachen. ${ }^{37}$ Pflichtige, die so wenig verdienen, dass sie keine Steuern zahlen, profitieren überhaupt nicht von der derzeitigen staatlichen Spendenförderung. Das BStGB setzt an Stelle der steuerlichen Förderung einen staatlichen Zuschuss zur Spende direkt an den Spendenempfänger. Der Zuschuss soll ein Drittel der jeweiligen Zuwendung betragen und direkt an den Zuwendungsempfänger ausbezahlt werden. Der Zuschuss ist somit eine typisierte und pauschalierte Steuerentlastung: Ob der Zuwender 100 Euro an die gemeinnützige Einrichtung spendet und bei einem Steuersatz von $25 \%$ in Höhe von 25 Euro entlastet wird, oder ob der Zuwender 75 Euro gibt und die gemeinnützige Einrichtung einen staatlichen Zuschuss von 25 Euro erhält, macht im Ergebnis keinen Unterschied. ${ }^{38}$

In einem solchen System wird jede Spende gleich gefördert, mag sie von einem einkommenstarken oder einem einkommenschwachen Steuerpflichtigen geleistet werden. Dieses System ist transparent. Der Spender kann sich auf den Zuschuss einstellen und seiner bisher übliche Spende um $25 \%$ reduzieren. Durch den staatlichen Zuschuss erhält die bedachte Organisation dennoch die gleiche Summe wie vor der Systemumstellung. Sie braucht keine Spendenquittungen mehr auszustellen. Der Spender muss keine Spendenquittungen mehr sammeln und seiner Steuererklärung beifügen.

\section{Neues Sanktionssystem}

Das BStGB führt ein neues System ein, steuerpflichtwidriges Verhalten zu sanktionieren. Dadurch soll das Steuerrecht entkriminalisiert und die Kooperation zwischen Verwaltung und Bürger vereinfacht werden. Die drei Grundtatbestände dieses Systems sind die steuerliche Haftung, der Verwaltungszuschlag und das Steuerstrafrecht.

Das Haftungsrecht eröffnet der Finanzbehörde die Möglichkeit, für ihre Ansprüche aus dem Steuerschuldverhältnis Dritte in Anspruch zu nehmen. Eine steuerliche Haftung soll nur den treffen, der zurechenbar daran beteiligt ist, den Besteuerungsgegenstand eines anderen Steuerpflichtigen der in ihm begründeten

37 v. Auer, L./Kalusche, A.: Spendenaufkommen, Steueraufkommen und Staatsaufgaben: Eine empirische Analyse, in: Walz, W.R./v. Auer, L./v. Hippel, T. (Hg.): Spenden- und Gemeinnützigkeitsrecht in Europa, 2007, 14.

38 Kirchhof, P.: Einkommensteuergesetzbuch, Ein Vorschlag zur Reform der Einkommen- und Körperschaftssteuer, 2004, § 4 Rn. 7. 
gesetzlichen Besteuerung zu entziehen. Als Haftende kommen in Betracht der pflichtwidrig handelnde Vertreter oder Vermögensverwalter, der Beteiligte an einer Steuerhinterziehung und der Entrichtungspflichtige, der eine Steuer nicht einbehält oder abführt.

Die steuerliche Haftung wird im BStGB durch ein (steuer)gesetzliches Pfandrecht ergänzt, das die Sachhaftung nach $\$ 76$ AO ersetzt. Da eine Ware keinen Besteuerungsgegenstand seiner Besteuerung entziehen, als Sache nicht steuerpflichtig und auch sonst nicht Träger von Rechten und Pflichten sein kann, ${ }^{39}$ befriedigt ein Pfandrecht und kein Haftungstatbestand im Bereich der Verbrauchsteuer das Sicherungsbedürfnis der Finanzverwaltung.

Als weiteres Element des Sanktionssystems sieht das BStGB bei Verwaltungsunrecht die Erhebung eines Verwaltungszuschlags vor. Die Tatbestände der einzelnen Handlungen oder Unterlassungen, die als Verwaltungsunrecht mit einem Verwaltungszuschlag belegt werden können, sind in den einzelnen Büchern gesetzlich geregelt. Diese Tatbestände betreffen die Verletzung eher formaler Pflichten.

Drittes Element des Sanktionssystems ist auch im BStGB das Steuerstrafrecht. Das öffentliche Interesse am vollständigen und rechtzeitigen Steueraufkommen und die Gleichheit vor dem Steuergesetz aus Art. 3 Abs. 1 GG fordern von der Finanzverwaltung, beim Vollzug der Steuergesetze das Interesse der rechtstreuen Steuerschuldner gegenüber denen zu wahren, die sich der ihnen gesetzlich zugemessenen Steuerlast auf widerrechtliche Weise zu entziehen suchen. ${ }^{40}$ Wie im geltenden Recht bedroht auch das BStGB ein derartiges Verhalten als Steuerhinterziehung mit Strafe. Das BStGB verzichtet auf Ordnungswidrigkeitstatbestände. Stattdessen ist zu prüfen, ob ein Verwaltungszuschlag in Betracht kommt.

Der Tatbestand einer Steuerhinterziehung im besonders schweren Fall entfällt. Wenn der Richter den Strafrahmen der Steuerhinterziehung nutzt, kann er regelmäßig den Unwertgehalt der Tat vollständig sühnen. Soweit sich hierdurch Unterschiede zur Strafdrohung des Betruges im besonders schweren Fall ( $\$ 263$ Abs. 3 StGB) und zum besonders schweren Fall des Diebstahls (§ 243 StGB) ergeben, ist zu erwägen, diese Vermögensdelikte ebenfalls mit dem Strafrahmen

39 Vgl. Loose, M., in: Tipke, K./Kruse, H.W.: Abgabenordnung, 2007, § 76, Rn. 2.

40 Joecks, W., in: Franzen, K./Gast, B./Joecks, W.: Steuerstrafrecht, 2009, 7. Auflage, Einleitung, Rn. 9; vgl. zum verfassungsrechtlichen Gebot, die steuerliche Belastungsgleichheit auch durch die Gestaltung des Erhebungsverfahrens zu sichern, BVerfGE 84, 239 - Kapitalertragsteuer. 
einer Freiheitsstrafe von bis zu fünf Jahren zu sühnen. Wünschenswert wäre ein allgemeiner Maßstab für Vermögensdelikte im StGB und im Nebenstrafrecht.

Das BStGB sieht einen persönlichen Strafaufhebungsgrund für geringfügige Steuerhinterziehung vor. Stattdessen verdoppelt sich die Steuerschuld. Das Bedürfnis der Rechtsgemeinschaft nach einer Sanktion wird auch so angemessen befriedigt.

Darüber hinaus sieht auch das BStGB die Möglichkeit einer strafbefreienden und auch einer strafmildernden Selbstanzeige vor. Es bietet dem Steuerhinterzieher die Möglichkeit, straffrei oder zumindest zu einer deutlich verminderten Strafe den Weg des Unrechts zu verlassen und zur Steuerehrlichkeit zurückzukehren. ${ }^{41}$ Die Finanzbehörde kann gleichzeitig bisher verheimlichte Steuerquellen erschließen ${ }^{42}$ und einen ansonsten häufig verlorenen Steueranspruch noch realisieren. ${ }^{43}$ Auch viele ausländische Rechtsordnungen sehen im Steuerstrafrecht Straffreiheit oder Strafmilderung bei einer Selbstanzeige des Täters vor. ${ }^{44}$

\section{Einkommensteuer}

Das deutsche Ertragsteuerrecht in seiner heutigen Gestalt bedarf dringend der Reform. Die geltenden Bestimmungen leiden an einem Übermaß an Detailregelungen, sind oft unverständlich und widersprüchlich. ${ }^{45}$ Das rechtfertigende Belastungsprinzip ist durch Subventions- und Lenkungstatbestände verdeckt. Hinzu treten strukturelle, systematische und sprachliche Mängel. Der Steuerpflichtige kann seine Steuererklärung aus eigenem Rechtsverständnis nicht mehr abgeben, die Richtigkeit seiner Erklärung nicht mit seiner Unterschrift verantworten. Das rechtsstaatliche Gebot der Voraussehbarkeit und Berechenbarkeit der Steuerlasten fordert einfache und klare einkommensteuerrechtliche Regelungen, die dem Adressaten verständlich sind. ${ }^{46}$

41 BGH, Urteil vom 20.5.2010 - 1 StR 577/09, DStR 2010, 1133.

42 BGH, Urteil vom 20.5.2010 - 1 StR 577/09, DStR 2010, 1133 m.w.N.

43 Vgl. zu Rechtfertigung und Zweck der Selbstanzeige nach $\S 371$ AO, Joecks, W., in: Franzen, K./Gast, B./Joecks, W., a.a.O., § 371, Rn. 19.

44 Vgl. hierzu Abramowski, J.: Die strafbefreiende Selbstanzeige ( $\$ 371$ AO) im internationalen Vergleich, DStZ 1992, 300 ff, ders., Verfassungswidrigkeit des steuerlichen Selbstanzeigeprivilegs?, in: DStZ 1992, $460 \mathrm{ff}$.

45 Vgl. BVerfGE 84, 239 (271) - Zinsbesteuerung.

46 BVerfGE 99, 216 (243) - Familienlastenausgleich II; Kirchhof, P.: Verfassungsrechtliche und steuersystematische Grundlagen der Einkommenssteuer, DStJG 24 (2001), 9 (20). 


\section{Besteuerung des Markteinkommens}

Ein Staat, der strukturell auf Staatsunternehmen verzichtet, die Produktionsfaktoren Arbeit und Kapital in privater Hand belässt, ist auf die Teilhabe am Erfolg privaten Wirtschaftens zur Finanzierung seiner eigenen Aufgaben angewiesen. Die Steuer ist daher Folge und Preis der Eigentümer- und Berufsfreiheit. ${ }^{47}$ Der Staat beansprucht seinen Anteil am Erfolg der individuell genutzten Freiheit. ${ }^{48}$ Er finanziert sich durch Steuern, die an die Leistungsfähigkeit anknüpfen, die der Steuerpflichtige durch Nutzung der von der Rechtsgemeinschaft bereitgestellten Erwerbsmöglichkeiten gewonnen hat. ${ }^{49}$

Steuerobjekt der Einkommensteuer ist das Einkommen, das nach dem Jahreseinkommensprinzip erfasst wird. Maßstab für die Ermittlung der Leistungsfähigkeit ist die Vermögensmehrung, die aus Erwerbshandeln, also der Nutzung von Erwerbsgrundlagen zur Erzielung von Einkünften am Markt erzielt wird. Das Jahressteuerprinzip sichert die Besteuerung des am gegenwärtigen Markt erwirtschafteten Einkommens. ${ }^{50}$ Das Entstehen stiller Reserven wird möglichst vermieden, Aufwand wirkungsgerecht zugeordnet.

\section{Befreiung von Lenkungsnormen}

Lenkungs- und Subventionsnormen, die in Steuergesetzen „versteckt“ sind, entziehen sich der Kontrolle von Parlament und Öffentlichkeit. Will der Staat fördernd leisten, sind hierfür besondere Leistungsgesetze geeignet. Die Steuersubvention durchbricht die Allgemeinheit der Steuer. Sie begünstigt erwünschtes und benachteiligt unerwünschtes Verhalten. ${ }^{51}$ Diese Steuersubvention ist zwar zulässig, darf aber nur nach bestimmten verfassungsrechtlichen Vorgaben vom Gesetzgeber angeboten werden. Das BStGB sucht das Einkommensteuerrecht auf seine Regeltatbestände zurückzuführen, auf Lenkungs- und Durchbrechungsnormen zu verzichten und dadurch den steuerlichen Belastungsgrund gleichheitsgerecht zu gestalten und für jedermann sichtbar zu machen. Das durch Weg-

Kirchhof, P., in: HStR, Band V, 2007,3. Aufl., § 118 Rn. 2.

48 ders.: Der Karlsruher Entwurf und seine Entwicklung zu einer Vereinheitlichen Ertragssteuer, in: StuW 2002, 3 (5).

49 ders.: Das EStGB - Ein Vorschlag zur Reform des Ertragssteuerrechts, in: DStR 2003, Beihefter 5 zu Heft $37,1$.

50 ders.: EStG, 9. Aufl., § 2 Rn. 18.

51 ders., in: HStR, a.a.O., § 118 Rn. 25. 
fall der Ausnahmetatbestände erzielte Mehraufkommen wird durch einen niedrigen Steuersatz aufkommensneutral an die Allgemeinheit zurückgegeben. ${ }^{52}$

\section{Nur noch eine Einkunftsart}

Wer einen Euro Einkommen erzielt, mehrt in jeder Einkunftsart seine Leistungsfähigkeit um diesen Wert. Deswegen behandelt das BStGB alle Einkünfte als gleichwertig und unterscheidet nicht mehr zwischen verschiedenen Einkunftsarten. Werden die Einkünfte in einem einzigen Grundtatbestand erfasst, stellt dies die gleiche Belastung aller Einkünfte sicher. Der Verzicht auf unterschiedliche Einkunftsarten vermeidet die darin angelegten Belastungsunterschiede, vereinfacht die Steuer und beseitigt ungerechtfertigte Differenzierungen. Der Einkommensteuer unterliegen alle Einkünfte aus Erwerbshandeln. Sondertatbestände entfallen. Tatbestandsunterscheidungen, etwa bei der Gewinnermittlung oder beim Quellenabzug, greifen erst dort, wo sie gerechtfertigt sind.

\section{Ein einheitlicher Steuersatz}

Das BStGB hält an einer Entlastung der Anfangseinkommen durch Progression fest, verlegt diese jedoch in die Bemessungsgrundlage der Einkommensteuer. Ein progressiver Tarif ist Ausdruck der vertikalen Steuergerechtigkeit, ${ }^{53}$ entlastet einkommensschwächere Schichten, soll die Belastungswirkung mit wachsendem Einkommen ansteigen, Steuer in diesem Maße ,spürbar“ bleiben lassen. ${ }^{54}$

Ein progressiver Tarif muss sich vor der Freiheitsgarantie rechtfertigen, dem Recht, sich von anderen unterscheiden und vorhandene Unterschiede mehren zu dürfen. ${ }^{55}$ Das Prinzip eines sozialen Ausgleichs rechtfertigt die Entlastung der Anfangseinkommen, kaum aber eine Progression als Grundprinzip für alle Einkommen. Das geltende Recht hat dieses Prinzip inzwischen aufgegeben. Der Steuersatz bei der Körperschaftsteuer beträgt 15\%, bei der Abgeltungssteuer für Einkünfte aus Kapitalvermögen 25\%, im Übrigen bei Einkommen von mehr als 8.004 bis 52.881 Euro pro Jahr $15 \%$ bis $42 \%$, ab 52.882 Euro linear $42 \%$, ab

52 Nach Feststellung des Kieler Instituts für Weltwirtschaft ist die vorgeschlagene Einkommensteuerreform aufkommensneutral, siehe Boss, A./Elendner, T.: Kieler Arbeitspapier Nr. 1205, 2004, Vorschläge zur Steuerreform in Deutschland: Was bedeuten sie? Was „,kosten“ sie?, 23, abzurufen unter www.ifwkiel.de.

53 Tipke, K.: Die Steuerrechtsordnung, a.a.O., 411.

54 Vgl. Becker, H.: Steuerprogression und Steuergerechtigkeit, in: Festschrift für Franz Klein, 382.

55 Kirchhof, P.: Das EStGB, a.a.O., Beihefter 5 zu Heft 37, 1 (9). 
250.731 Euro linear 45\%. Das Zusammenwirken von Einkommensteuer und Gewerbesteuer macht die Steuer teilweise zu einem Instrument des Finanzausgleichs. ${ }^{56}$ Der progressive Tarif verkompliziert die Steuer, zieht Folgewirkungen wie den Splitting-Tarif ( $\S 26 \mathrm{ff}$. EStG) oder den Progressionsvorbehalt ( $\$ 32 \mathrm{~b}$ $\mathrm{EStG}$ ) nach sich, zeigt sich hierdurch empfänglich für immer neue Steuergestaltungen und gesetzgeberische Korrekturen. Er schafft Anreiz, eine höhere Progressionsstufe durch eine Ausweichbewegung zu verhindern. In seinen Auswirkungen ist der progressive Tarif nur schwer verständlich und kaum mehr vermittelbar.

Das BStGB vermeidet diese Nachteile, indem es formal einen einheitlichen Steuersatz vorsieht, die Progression aber in der Bemessungsgrundlage, dort in einem sozialen Ausgleich berücksichtigt. Jedem unbeschränkt Steuerpflichtigen steht sein Existenzminimum in Form des Grundfreibetrags von 8.000 Euro zu. Zudem wird eine Vereinfachungspauschale von 2.000 Euro für Erwerbskosten von den Erwerbserlösen abgezogen. Die folgenden 5.000 Euro werden nur zu 60 $\%$, die weiteren 5.000 Euro zu $80 \%$ besteuert. Die volle Steuerpflicht setzt deshalb erst bei einem Einkommen von 20.001 Euro ein. Der Abzug der sozialen Ausgleichsbeträge bewirkt trotz des linearen Steuersatzes eine Progression.

Der einheitliche Steuersatz stellt Gleichheit für alle Steuerpflichtigen sicher. Aktuell unterschiedliche Steuersätze in Körperschaftsteuer und Einkommensteuer verhindern eine rechtsformneutrale Besteuerung und veranlassen wirtschaftliche und gesellschaftsrechtliche Ausweichgestaltungen, wie die GmbH \& Co. KG. Gilt dagegen ein einheitlicher Steuersatz, können viele Einkünfte abschlieBend an der Quelle besteuert werden. Das gilt für Arbeitslohn, für Kapitaleinkünfte, für Alterseinkünfte und für Einkünfte aus einer Beteiligung. Eine Anrechnung vorausgezahlter Steuer und das Teileinkünfteverfahren erübrigen sich. Das Recht gewinnt an Einfachheit, Klarheit und Verständlichkeit.

\section{Integration der Körperschaftsteuer in die Einkommensteuer}

Das BStGB besteuert Personen- und Kapitalgesellschaften als steuerjuristische Person gleich. Die Trennung zwischen Körperschaftsteuer und Einkommensteuer wird aufgegeben, die Ungleichbehandlung zwischen der Anwendung eines progressiven Tarifs der Einkommensteuer beim Anteilseigner und eines linearen

56 Bareis, P.: Die Reform der Einkommenssteuer vor dem Hintergrund der Tarifentwicklung seit 1934, in: Festschrift Klaus Offerhaus, 1053 (1063). 
Tarifs von $15 \%$ bei der Körperschaft wird beendet. Die steuerjuristische Person umfasst jeden wirtschaftlich selbstständigen Organismus, an dem mehrere Personen beteiligt sind, also sämtliche Personengesellschaften und die bisher Körperschaftsteuerpflichtigen. Die Körperschaftsteuer wird überflüssig. Die von der steuerjuristischen Person erwirtschafteten Einkünfte werden bei ihr abschließend besteuert. Die Weiterleitung von Einkünften an die Beteiligten bleibt steuerfrei.

Grundlage dieser Vorausbesteuerung ist der einheitliche Steuersatz. Werden Wertschöpfungen bei der steuerjuristischen Person endgültig besteuert und gilt für die Anteilseigner derselbe Steuersatz, bedarf es keiner Korrektur, wenn die vorbesteuerten Erträge an die Beteiligten weitergeleitet werden. Die Weiterleitung schöpft keinen neuen Wert, verteilt lediglich die von der steuerjuristischen Person selbst erwirtschafteten Erträge. Der Verwaltungsaufwand verringert sich. Einheitlich und gesondert festgestellte Besteuerungsgrundlagen auf der Ebene der Gesellschaft entfallen. Die steuerjuristische Person selbst ist Steuersubjekt. Sie gibt Steueranmeldungen ab und wird selbständig veranlagt.

Werden die Erträge bei der steuerjuristischen Person abschließend besteuert, wirkt sich dies auch auf die Beurteilung von Erlösen aus der Veräußerung von Anteilen an steuerjuristischen Personen aus. Veräußert ein Anteilseigner seine Beteiligung, erzielt er einen umso höheren Veräußerungspreis, je größer das Kapital der steuerjuristischen Person ist. Thesauriert die steuerjuristische Person Gewinne, realisiert sich im Veräußerungspreis ein Entgelt für die realisierten und vorab bereits besteuerten Erträge des Unternehmens, aber auch für verbliebene stille Reserven und Erwartungen auf zukünftige Gewinne. Veräußerungsgewinne dürfen daher nicht aufgrund der Vorbesteuerung insgesamt unbesteuert bleiben. Das BStGB schätzt typisierend und widerlegbar die Veräußerungskosten mit neun Zehnteln des zugeflossenen Erlöses, besteuert dementsprechend $10 \%$ des Veräußerungspreises.

\section{Familiengerechte Besteuerung}

Das Existenzminimum bleibt von der Besteuerung ausgenommen. ${ }^{57}$ Würde der Staat das existenznotwendige Einkommen besteuern, so würde er die Funktion der Eigentumsgarantie, dem Eigentümer einen Freiraum im vermögensrechtlichen Bereich zu sichern und ihm dadurch eine eigenverantwortliche Gestaltung 
zu ermöglichen, ${ }^{58}$ im Elementaren verfehlen, ihm außerdem einen Anspruch auf staatliche Sozialhilfe gewähren müssen. Das vom Grundrechtsberechtigten zur Bestreitung seines notwendigen Lebensunterhalts und - unter Berücksichtigung von Art. 6 Abs. 1 GG - desjenigen seiner Familie benötigte Einkommen (Existenzminimum) ist deshalb für eine Besteuerung schlechthin nicht verfügbar. ${ }^{59}$ Das BStGB bemisst das Existenzminimum nach den allgemeinen wirtschaftlichen Verhältnissen und dem in der Rechtsgemeinschaft anerkannten Mindestbedarf typisierend auf einen Grundfreibetrag in Höhe von 8.000 Euro.

Art. 6 Abs. 1 GG schützt die Vereinigung der Ehe und Familie besonders, weil dem Staat und seiner Rechtsordnung an diesen Gemeinschaften um seiner Zukunft willen besonders gelegen ist. Das Steuerrecht muss die Ehe als Erwerbsgemeinschaft ${ }^{60}$ und die Familie als Unterhaltsgemeinschaft anerkennen. Nicht dauernd getrennt lebende Ehegatten bestreiten ihren Lebensunterhalt regelmäßig gemeinsam. Das Erwirtschaftete steht beiden zu gleichen Teilen zu. ${ }^{61}$ Deswegen ermöglicht das BStGB, dass Grundfreibetrag und Sozialausgleichsbetrag unter den Ehegatten übertragen werden können. Ebenso können Verluste innerhalb der ehelichen Erwerbsgemeinschaft ausgeglichen werden. Das Einkommensteuerrecht bietet damit für Ehegatten ähnliche Steuerregeln, wie sie für andere Erwerbsgemeinschaften, wie etwa bei der OHG oder der $\mathrm{GmbH}$, gelten. Dort erscheint es selbstverständlich, dass der erzielte Gewinn unter den Gesellschaftern aufteilbar ist und entsprechend der Aufteilung versteuert werden kann.

Die Familie bildet eine Unterhaltsgemeinschaft. Das Einkommensteuerrecht erfasst das Kind als Unterhaltsberechtigten, der die steuerliche Leistungsfähigkeit der unterhaltspflichtigen Eltern mindert. Da die Eltern einen Teil ihres Einkommens als Unterhaltsleistungen an die Kinder weitergeben müssen, können sie über diesen Einkommensbetrag nicht anderweitig verfügen; ihre steuerbare Leistungsfähigkeit ist insoweit verringert. ${ }^{62}$ Der Kindesbedarf ist daher steuerlich durch Kindergeld oder Kinderfreibeträge zu berücksichtigen. ${ }^{63}$ Das BStGB hat sich für die Kindergeldlösung entschieden. Das Kindergeld selbst bleibt steuer-

59 BVerfGE 87, 153 (169f.) - Grundfreibetrag; BVerfGE 112, 268 (280) - Berufsbedingte Kinderbetreuungskosten.

60 BVerfGE 61, 319 (342 ff.) - Ehegattensplitting.

61 BVerfGE 61, 319 (347) - Ehegattensplitting.

62 BVerfGE 82, 60 (86f.) - Familienexistenzminimum; BVerfGE 99, 246 (259f.) - Familienleistungsausgleich. BVerfGE 112, 164 (174f.) - Familienbesteuerung. 
frei. Gesetzlich Unterhaltsberechtigte, für die kein Anspruch auf Kindergeld besteht, können ihren Grundfreibetrag auf den Unterhaltspflichtigen übertragen, nicht aber den Sozialausgleichsbetrag.

\section{Nachgelagerte Besteuerung}

Gelder, die zur persönlichen Zukunftssicherung geleistet werden, stehen für den gegenwärtigen Bedarf nicht zur Verfügung. Erst die spätere Zahlung der Versicherungsträger verschafft dem Begünstigten wirtschaftliche Leistungsfähigkeit. Verlangt der Staat, dass eine individuelle Vorsorge zu treffen ist, verbietet sich die vorgelagerte Besteuerung der Zukunftssicherungsleistungen. ${ }^{64}$ Deswegen stellt das BStGB Erwerbserlöse, die für Beiträge der eigenen Zukunftssicherung verwendet werden, in der Ansparphase steuerfrei. Die in der Auszahlungsphase zufließenden Rentenerlöse sind dann zu versteuerndes Einkommen. Eine Doppelbelastung des verwendeten Einkommens wird vermieden.

\section{Weniger Veranlagungen}

Das BStGB erhebt die Steuer entweder durch einen grundsätzlich abschließenden Quellenabzug oder durch Veranlagung. Bei Erwerbshandeln aus abhängiger Lohnarbeit, aus Überlassung von Geldvermögen und aus der Altersversorgung behält die auszahlende Stelle als Entrichtungspflichtiger die Einkommensteuer ein und führt sie an die zuständige Finanzbehörde ab. Aufwendungen lassen sich meist durch die Vereinfachungspauschale typisieren. Deswegen erübrigt sich in der Regel eine nachträgliche Veranlagung.

Einkünfte, die nicht an der Quelle zu besteuern sind, werden veranlagt. Das BStGB sieht die Selbstveranlagung als Regelmethode vor. Der Steuerpflichtige berechnet die Steuer selbst auf einem amtlichen Vordruck und meldet die Steuer an. Mit der Selbstveranlagung erlebt der Steuerpflichtige die steuerlichen Zusammenhänge zwischen der Einkunftserzielung aus Erwerbshandeln und der daraus entstandenen Steuer, kann sein zukünftiges Erwerbshandeln an dieser gesicherten Grundlage ausrichten. 


\section{Gemeindezuschlag}

Der Reformvorschlag löst die Gewerbesteuer durch eine kommunale Zuschlagsteuer zur Einkommensteuer ab. Der Zuschlag wird auf die reformierte Einkommensteuer erhoben, in die auch die Körperschaftsteuer integriert ist.

Diese Gemeindeertragsteuer beteiligt alle Wirtschaftssubjekte, die in einer Gemeinde Einkommen erzielen, an den Kosten für die Bereitstellung kommunaler Infrastruktur, unabhängig von Beruf, Einkunftsart und Rechtsform. Die Steuer ist für alle spürbar. Mit Ausweitung des Kreises der Steuerpflichtigen muss die Hebesatzentscheidung vor einem größeren Kreis von Betroffenen verantwortet werden. Sie macht Belastungsentscheidungen transparent. Der Bürger erlebt Kommunalpolitik in Selbstbetroffenheit. Die Entscheidung über die Hebesätze wird demokratisch vor Ort legitimiert.

Ein Ersatz der Gewerbesteuer durch Zuschläge bei der Einkommensteuer stattet Städte und Umlandgemeinden angemessen mit Finanzkraft aus. Die Ertragswirkungen der Gemeindeertragsteuer können aufgabengerecht auf die Gemeinden verteilt werden. Eine Betriebs- oder Wohnsitzverlegung, die Steuern sparen soll, lohnt sich nicht, weil sich Steuerberechtigung und Hebesatz nach dem Ort des jeweiligen Erwerbshandelns richtet. Die Bemessungsgrundlage ist wesentlich verbreitert und weniger konjunkturempfindlich. Die Gemeinden erhalten mit der Gemeindeertragsteuer eine erneuerte stabilere Grundlage ihrer Selbstverwaltungsautonomie.

\section{Schwächen des geltenden Gewerbesteuerrechts}

Eine große Anzahl von Unternehmen wird trotz ihrer Nähe zu den Freiberuflern und sonstigen Selbständigen unter dem Sammelbegriff „Gewerbetreibende” erfasst und mit Gewerbesteuer belastet. ${ }^{65}$ Dieser Tatbestand des Gewerbebetriebs überzeugt als ein die Steuerpflicht begründendes Merkmal nicht: ${ }^{66}$ Der Makler, der mit einem Computer und einem Telefon arbeitet und die kommunale Infrastruktur kaum in Anspruch nimmt, ist Gewerbetreibender und unterliegt der Gewerbesteuer, der Arzt mit vielen Mitarbeitern, Geräten und Patienten ist Freiberufler. Wird eine Zahnprothese in der Werkstatt eines Zahntechnikermeisters

$65 K e \beta, T .:$ Anmerkung zum Beschluss des BVerfG vom 15.1.2008 - 1 BvL 2/04, BVerfGE 120, 1, in: FR $2008,829$.

66 Zur Abgrenzung des Gewerbebetriebs von der Land- und Forstwirtschaft, vgl. BFH, Urteil vom 8.11.2007 - IV R 35/06 und Urteil vom 25.3.2009 - IV R 21/06; zur Abgrenzung von der selbständigen Tätigkeit vgl. die Übersicht über die Einzelentscheidungen in H 15.6 EStR. 
hergestellt, gilt dies als Gewerbe. ${ }^{67}$ Wird die Prothese in der Praxis eines Zahnarztes von einem angestellten Zahntechniker hergestellt, gilt dies als Nebenbetrieb der Zahnarztpraxis und unterliegt nicht der Gewerbesteuer. ${ }^{68}$

Die schwierige Abgrenzung zwischen einer gewerblichen und einer freiberuflichen oder land- und forstwirtschaftlichen Tätigkeit führt auch zu Unsicherheit und Streitfällen. Die Tätigkeit von Rechtsanwälten als Insolvenzverwalter wird als eine sonstige selbständige (vermögensverwaltende) Tätigkeit qualifiziert, ${ }^{69}$ ein selbständiger Buchhalter, der eigenverantwortlich Steuererklärungen, Einnahme-Überschuss-Rechnungen und Jahresabschlüsse als freier Mitarbeiter für Steuerberater erstellt, übt keinen einem Steuerberater oder Steuerbevollmächtigten ähnlichen Beruf aus. ${ }^{70}$ Ein Ingenieur, der als Architekt arbeitet, aber nicht nur Architektenleistungen erbringt ist - zumindest auch - gewerblich tätig, ${ }^{71}$ eine Personengesellschaft erzielt insgesamt gewerbliche Einkünfte, wenn sich eine so genannte Freiberufler-Kapitalgesellschaft mitunternehmerisch an einer Freiberufler-Personengesellschaft beteiligt. ${ }^{72}$ Eine Personengesellschaft hingegen, die sich aus Angehörigen unterschiedlicher freier Berufe zusammensetzt, ist grundsätzlich nicht als gewerbliche Mitunternehmerschaft einzustufen. ${ }^{73}$

Durch die Einführung der Gewerbesteuerumlage, ${ }^{74}$ den Wegfall der Lohnsumme als Besteuerungsgrundlage ${ }^{75}$ und die Abschaffung der Gewerbekapitalsteuer zum $01.01 .1998^{76}$ hat sich die Gewerbesteuer von einer Objektsteuer zu einer Ertragsteuer entwickelt, sich damit von der ursprünglichen Grundstruktur einer Objektsteuer entfernt. Die heute allein an die Einkünfte aus Gewerbebetrieben anknüpfende Gewerbesteuer ist nicht mehr geeignet, den notwendigen Beitrag FG Köln, Urteil vom 28.5.2008 - 12 K 3735/05, EFG 2008, 1876, Revision beim BFH anhängig unter VIII R 29/08; vgl. dazu auch FG Hamburg, Urteil vom 27.5.2009 - 2 K 72/07, EFG 2009, 1651, Revision beim BFH anhängig unter VIII R 37/09.

70 BFH, Beschluss vom 23.1.2008 - VIII B 46/07, BFH/NV 2008, 785.

71 BFH, Urteil vom 18.10.2006 - XI R 10/06, BStB1. II 2008, 54. BFH, Urteil vom 23.11.2000 - IV R 48/99, BStB1. II 2001, 241.

74 Vgl. Art. 106 Abs. 5 und 6 GG i.d.F. vom 12.05.1969, BGBl. I 1969, 359 und $\S 6$ Gemeindefinanzreformgesetz vom 8.9.1969, BGB1. I 1969, 1587.

75 Vgl. Steueränderungsgesetz 1979, BGB1. I 1978, 1849.

76 Vgl. Art. 4 des Gesetzes zur Fortsetzung der Unternehmenssteuerreform, BGB1. I 1997, 2590. 
zur Finanzierung der Gemeinden zu leisten. ${ }^{77}$ Der gewerbliche Gewinn ist stark von konjunkturellen Schwankungen, manche Gemeinde finanziell vom schwankenden Erfolg eines einzigen Großbetriebs abhängig. Die Steuerausfälle bei der Gewerbesteuer und der Wunsch nach einer „Objektivierung“ der Steuer haben den Gesetzgeber dazu verleitet, die Ausfälle durch unsystematische Erweiterungen der gewerbesteuerlichen Bemessungsgrundlage zu kompensieren, insbesondere durch die Hinzurechnung des $\S 8$ Nr. 1 a) GewStG, der ein Viertel des Entgelts für Schulden dem Gewinn aus Gewerbebetrieb hinzurechnet.

Die Gewerbesteuer leidet auch an ihrer mangelnden Rechtsformneutralität. Die Folgen zeigen sich vor allem in der als gleichheitswidrig empfundenen „Abfärberegelung“ des $\S 15$ Abs. 3 Nr. 1 EStG, wonach die Tätigkeit einer Personengesellschaft als insgesamt gewerblich gilt, wenn nur ein kleiner Teil tatsächlich gewerblich ist. ${ }^{78}$ Die Vorschrift veranlasst gesellschaftsrechtliche Gestaltungen, um die „Abfärberegelung“ zu umgehen.

Gleichzeitig hat der Gesetzgeber mit $§ 35$ EStG versucht, die ungleiche steuerliche Belastung zwischen Einzelunternehmen und Personengesellschaften auf der einen Seite und Kapitalgesellschaften auf der anderen Seite dadurch zu vermeiden, dass die Gewerbesteuer auf die Einkommensteuer angerechnet wird. Diese unsystematische Lösung führt vor allem durch die Ausgestaltung der Anrechnung zu Über- und Unterkompensationen der Steuer.

Eine Steuer soll dem Staat Einnahmen verschaffen. Die Anrechnung der Gewerbesteuer auf die Einkommensteuer nach $\S 35 \mathrm{EStG}$ läuft diesem Ziel zuwider. Diese Teilanrechnung verlagert das Steueraufkommen entgegen der Vorgaben des Art. 106 GG zwischen den Gebietskörperschaften und führt dazu, dass sich die Gewerbesteuer von einer steuerlichen Belastung in eine besondere Form des Finanzausgleichs verwandelt, da die Gewerbesteuer überwiegend den Gemeinden und die Einkommensteuer in erster Linie dem Bund und den Ländern zufließt. ${ }^{79}$ Gleiches gilt für eine Körperschaftsteuer, die sich wegen der hinzutretenden Gewerbesteuer mit einem Steuersatz von $15 \%$ begnügt.

77 Hey, J.: Kommunale Einkommen- und Körperschaftssteuer, zugleich ein Beitrag zur Bedeutung des Äquivalenzprinzips für die Ausgestaltung kommunaler Steuern, in: StuW 2002, 314 (315).

78 Zum Versuch eines „Gegensteuerns“ durch restriktive Auslegung vgl. BFH, Urteil vom 30.8.2001 - IV R 43/00, BStB1. II 2002, 152; Urteil vom 6.10.2004 - IX R 53/01, BStB1. II 2005, 383; Urteil vom 28.6.2006 - XI R 31/05, BStB1. II 2007, 378.

79 Schön, W.: Zum Entwurf eines Steuersenkungsgesetzes, in: StuW 2000, 151 (156). 


\section{Hebesätze bei der erneuerten Einkommensteuer}

Das kommunale Hebesatzrecht vereinfacht das Steuersystem und besteuert gleichheitsgerecht nach der Leistungsfähigkeit, unabhängig von der Rechtsform und der Art der Einkünfte:

- In der erneuerten Einkommensteuer gibt es nicht mehr sieben, sondern nur noch eine Einkunftsart. Alle Einkünfte werden gleich behandelt.

- Die Bemessungsgrundlage ist von Lenkungs- und Durchbrechungsnormen bereinigt. Der gleichheitsgerecht gestaltete Belastungsgrund wird wieder sichtbar.

- Alle rechtlich fassbaren Organismen bilden eine steuerjuristische Person, in der ein einheitlicher Gewinn erklärt, die Steuer ermittelt, festgestellt und bezahlt wird; so ist die Gleichbehandlung von Mitunternehmerschaften und Körperschaften gewährleistet.

- Es gibt keine Ausnahmen. Die Steuerlast muss von allen Steuerpflichtigen nach Maßgabe ihrer Leistungsfähigkeit getragen und kann nicht umgangen werden.

Durch den Verzicht auf Steuervergünstigungen und Ausnahmeregelungen erweitert die bereinigte Einkommensteuer die Bemessungsgrundlage im Vergleich zur heutigen Ertragsteuer erheblich und bietet schon aufgrund ihrer Ertragsstärke eine geeignete Grundlage für eine kommunale Zuschlagsteuer. Die Bemessungsgrundlage ist so breit, dass bereits geringe Hebesätze genügen, um die Gemeinden mit ausreichenden Finanzmitteln auszustatten. Die Einbeziehung der Einkünfte aller natürlichen und steuerjuristischen Personen ermöglicht eine Zuschlagsteuer ausnahmslos für alle Betriebe.

\section{Grundprinzipien der neuen Steuer}

Die Gemeindeertragsteuer ist eine wirtschaftskraftbezogene Steuerquelle im Sinne des Art. 28 Abs. 2 Satz 3 HS 2 GG. Sie knüpft an das in der jeweiligen Gemeinde erwirtschaftete Einkommen an, das auch Bemessungsgrundlage für die Einkommensteuer ist. ${ }^{80}$ Die Gemeinden erzielen Steuererträge, weil Steuerpflichtige in ihrem Gebiet wirtschaften und erfolgreich sind; mit ihrer Infrastruktur- und Hebesatzpolitik können sie diesen Erfolg beeinflussen. Steuerpflichtig

80 Siehe auch BVerfG, Beschluss vom 27.1.2010 - 2 BvR 2185/04 und 2 BvR 2189/04, Rn. 75, BFH/NV 2010, 793, wonach die Gewerbeertragsteuer und die Einkommensteuer die beiden einzigen ,wirtschaftskraftbezogenen Steuerquellen“ sind; so auch Scholz, R., in: Maunz, Th./Dürig, G. (Hg.), a.a.O, Art. 28, Rn. 84d. 
sind nicht nur Gewerbetreibende im herkömmlichen Sinn, sondern alle Steuersubjekte der vereinheitlichten Einkommensteuer.

So werden Gestaltungen ausgeschlossen, schwierige Abgrenzungsfragen im Hinblick auf die Gewerblichkeit vermieden. Steuerplanungskosten der Steuerpflichtigen und Vollzugskosten der Verwaltung können gering gehalten werden.

Vier Elemente stützen die kommunale Autonomie:

- Die Gemeinden können durch die Hebesatzentscheidung die Höhe der Steuereinnahmen selbst bestimmen.

- Das Hebesatzrecht knüpft wirtschaftskraftbezogen an den Ort des Erwerbshandelns an.

- Kommunale Anteile am Einkommensteuer- und Umsatzsteueraufkommen werden weiterhin entsprechend Art. 106 Abs. 5 und 5a GG verteilt.

- Die Steuerhoheit bleibt bei den Gemeinden.

Die Gemeinden sind verpflichtet, eine Gemeindeertragsteuer zu erheben. Auch das geltende Gewerbesteuerrecht sieht seit dem 01.01.2004 in $\S 16$ Abs. 4 Satz 2 GewStG einen Mindesthebesatz vor. ${ }^{81}$

\section{Verteilung der Steuererträge auf die Gemeinden}

Die Gemeindeertragsteuer belastet das in der Gemeinde erwirtschaftete Einkommen. Steuerberechtigt sind die Gemeinden, in der Steuerpflichtige durch Erwerbshandeln Einkünfte erzielen. Unternehmen erzielen ihre Einkünfte in der Gemeinde, in der sich die Betriebsstätte befindet. Arbeitnehmer erzielen ihren Arbeitslohn in der Gemeinde, in der sie arbeiten. Lässt sich der Ort des Erwerbshandelns - wie bei den privaten Kapitaleinkünften - nicht eindeutig bestimmen, ist an den Wohnsitz des Steuerpflichtigen anzuknüpfen. Unterhält ein Unternehmen Betriebsstätten in verschiedenen Gemeinden, ist der Unternehmensgewinn auf die Betriebsstättengemeinden nach der Zahl der beschäftigten Arbeitnehmer aufzuteilen. Dieser Schlüssel ist leicht feststellbar und bildet typisierend das Verhältnis ab, in welchem Betriebsstätten die örtliche Infrastruktur in Anspruch nehmen.

81 Im Beschluss vom 27.1.2010 - 2 BvR 2185/04 und 2 BvR 2189/04, BFH/NV 2010, 793, bestätigt das BVerfG die Verfassungsmäßigkeit des geltenden Mindesthebesatzes, ohne allerdings diesen von Verfassungs wegen zwingend vorauszusetzen. 


\section{Verwaltung und Verfahren}

Die Gemeindeertragsteuer will die finanzielle Eigenverantwortung stärken. Die Gemeinden allerdings wären angesichts der Vielzahl von Steuerpflichtigen und Zahlungsvorgängen überfordert, wenn sie die Gemeindeertragsteuer selbst verwalten sollten. Nach dem Vorbild der Kirchensteuer sieht der Gesetzesvorschlag daher eine Übertragung der Verwaltungskompetenz auf die Finanzbehörden gegen ein Verwaltungsentgelt vor. Da die Finanzbehörden für die Gemeinden tätig sind, haben die Gemeinden einen Anspruch, umfassend über das Ergebnis des Besteuerungsverfahrens informiert zu werden.

Die Gemeindeertragsteuer wird zusammen mit der Einkommensteuer erhoben und ist auch im Quellenbesteuerungsverfahren leicht zu verwalten. Arbeitnehmer und Unternehmen versteuern ihre Erträge in der Regel nach demselben Hebesatz, weil sich Betriebsstätte und Arbeitsplatz in derselben Gemeinde befinden. Selbst wenn ein Unternehmen Betriebsstätten in verschiedenen Gemeinden unterhält, bleibt die Zahl überschaubar.

\section{Keine Umlage}

Im Interesse der finanziellen Eigenverantwortung verzichtet der Vorschlag auf die bisherige Gewerbesteuerumlage. Das Aufkommen der Gemeindeertragsteuer soll bei den Gemeinden bleiben. Die geltende Gewerbesteuerumlage gemäß $\S 6$ Gemeindefinanzreformgesetz ist nicht gerechtfertigt, weil sie die Begründung der Gewerbesteuer als Äquivalent für kommunale Infrastrukturleistungen fragwürdig macht ${ }^{82}$ und die Finanzautonomie der Gemeinden erheblich schmälert. Von dem ursprünglich vereinnahmten Gewerbesteueraufkommen geht ein beträchtlicher Teil an den Bund und die Länder. ${ }^{83}$ Die Kommunen sind am Verfahren zur Änderung der Gewerbesteuerumlage nicht beteiligt. Im Zeitraum von 1993 bis heute waren sämtliche Änderungen der Gewerbesteuerumlage nicht durch Änderungen der Gewerbesteuer veranlasst, sondern dienten allein der Verteilung des Steueraufkommens.

82 Vgl. BVerfGE 46, 224, 236 f.; Montag, H., in: Tipke, K./Lang, J.: Steuerrecht, 2010, 20. Aufl., 468: „starke Relativierung des Äquivalenzprinzips“.

83 Im Jahr 2008 haben die Gemeinden brutto rund 41 Mrd. Euro Gewerbesteuer eingenommen. Davon flossen rund 6,7 Mrd. Euro in die Gewerbesteuerumlage, von der rund ein Viertel dem Bund und drei Viertel den Ländern zusteht, vgl. Statistisches Bundesamt: Statistisches Jahrbuch 2009, 579. 


\section{Umsatzsteuer}

\section{Mängel des geltenden Rechts}

Die Umsatzsteuer belastet den Verbraucher, ist also eine Verbrauchsteuer. ${ }^{84}$ Gegenwärtig werden dennoch alle Umsätze - auch die zwischen Unternehmern zunächst belastet, danach durch einen Vorsteuerabzug wieder entlastet. Dieses aufwändige Verfahren ist zu kompliziert und führt zu Liquiditätsverzerrungen, weil der leistende Unternehmer die Umsatzsteuer abführen muss, bevor er sie vereinnahmt hat und sein Leistungspartner die Vorsteuern erstattet erhält, bevor er sie bezahlt hat. Zudem drohen einzelne Ausgestaltungen und Ausnahmen die Rechtsstruktur der Umsatzsteuer - ihre Rechtlichkeit - zu verdrängen. Die Steuerbefreiungen folgen keiner Systematik, knüpfen an den Inhalt einer Leistung, den Leistenden ${ }^{85}$ oder den Leistungsempfänger ${ }^{86}$ an und sind in ihrer Vielfalt nicht mehr zu überschauen. Die Fallgestaltungen des ermäßigten Steuersatzes sind kaum voneinander abgrenzbar. Der Ausschluss des Vorsteuerabzugs bei Steuerfreiheit der Leistung führt zu Verwerfungen. ${ }^{87}$ Die steuerfreie innergemeinschaftliche Lieferung wird dem harmonisierten Binnenmarkt nicht gerecht. Zudem erschweren die vom Bestimmungslandprinzip abweichenden Regelungen zum Ort einer Leistung die Bestimmung des anwendbaren Rechts.

Die Besteuerung knüpft an die am Markt eingesetzte Kaufkraft, ${ }^{88}$ die Vermögensverwendung an. Diese grob typisierte, vermutete Leistungsfähigkeit des Verbrauchers belegt eine steuerlich nutzbare Finanzkraft. Das geltende Recht setzt diese Belastungsentscheidung nicht konsequent um, wenn es auch Leistungen an Unternehmer und an die öffentliche Hand besteuert.

Reformansätze, einzelne Regelungen zu vereinfachen, gibt es viele. ${ }^{89}$ Diese suchen vor allem die Betrugsanfälligkeit ${ }^{90}$ des Besteuerungssystems zu verringern.

84 Vgl. BVerfGE 31, 314 (331) - 2. Rundfunkentscheidung.

85 Vgl. § 4 Nr. 11b, 19 UStG.

86 Vgl. § 4 Nr. 17 Buchst. b UStG.

87 Vgl. auch BVerfG-Beschluss vom 29.8.2006 1 BvR 1673/06, UR 2007, 464; BFH-Urteil vom 9.11.2006 V R 43/04, UR 2007, 111; Kohlhaas, K.-F.: Abwehrstrategien zur Vermeidung der Umsatzsteuerfreiheit kultureller Veranstaltungen - Zur Gleichartigkeit der Einrichtung privater Unternehmen i.S. des $\S 4$ Nr. 20a UStG, in: DStR 2008, 1020.

88 Vgl. Kirchhof, P., HStR, a.a.O., § 118 Rn. 243.

89 „Reverse-Charge-Verfahren“, vgl. Zusammenfassender Ergebnisbericht der PSP-GmbH, November 2005; vgl. Europäische Kommission: Mitteilung der Kommission über mögliche Maßnahmen zur Bekämpfung von MwSt-Betrug vom 22.02.2008 KOM (2008) 109 endg. = UR 2008, 251; Vorstufenbefreiung, vgl. Mittler, G.: Einführung von Vorsteuerbefreiungen als Mittel zur UmsatzsteuerBetrugsbekämpfung, in: UR 2001, 385. 
Das BStGB führt die Umsatzbesteuerung auf ihre Grundprinzipien zurück, schafft Klarheit und Verständlichkeit und macht so die Besteuerung für jedermann wieder einsichtig. Es ist in einfacher Sprache verfasst, trifft keine Einzelfallentscheidungen, ist so formuliert, dass es nicht durch den Wandel des Wirtschaftslebens widerlegt wird, ist frei von Privilegien und Verfremdungen und so gestaltungsresistent.

\section{Leistung an Verbraucher, zwischenunternehmerische Leistungen}

Die geltende Allphasen-Nettoumsatzsteuer mit Vorsteuerabzug belastet jede Produktions- und Handelsstufe mit Umsatzsteuer. Der Unternehmer wird durch den Vorsteuerabzug wieder entlastet, das Steueraufkommen entsteht erst bei Leistungen an den Verbraucher. In diesem Verfahren wird deutlich mehr als die vierfache Summe des späteren Umsatzsteueraufkommens in Deutschland in Rechnung gestellt, bezahlt und dokumentiert. Im Jahr 2008 betrug die Gesamtsumme der in Rechnung gestellten und vom Leistungsempfänger gezahlten Umsatzsteuer vor Abzug der Vorsteuerbeträge 846 Mrd. Euro. Diese Summe führte aber nur zu einem Ertrag aus Umsatzsteuer - einschließlich Einfuhrumsatzsteuer $^{91}$ - von 177 Mrd. Euro. ${ }^{92}$ Die umsatzsteuerliche Entlastung durch den Vorsteuerabzug des geltenden Rechts veranlasst also ein „Nullsummenspiel“93 von erheblichem Ausmaß.

Diese Wertbewegungen sind anfällig für Betrug und gefährden das Steueraufkommen bei Insolvenz. Betrugsmöglichkeiten eröffnen sich, wenn Umsatzsteuern vereinnahmt, aber nicht an den Fiskus abgeführt oder ungerechtfertigte Vorsteuererstattungen verlangt werden. Bei Insolvenzen sind die staatlichen Steueransprüche gefährdet, wenn der Unternehmer Umsatzsteuer vereinnahmt, er jedoch vor Bezahlung an den Fiskus insolvent wird oder unberechtigte Vorsteuererstattungen nicht zurückzahlen kann.

Der Belastungsgrund der Umsatzsteuer ist die Kaufkraft des Verbrauchers. Die eingesetzte Kaufkraft wird im Leistungstausch sichtbar, im Entgelt zählbar. Die

90 Möglich ist die Vorsteuererschleichung, der Karussellbetrug oder das Nichtabführen der Umsatzsteuer.

91 Diese ist hier einzubeziehen, denn die gezahlte Einfuhrumsatzsteuer berechtigt zum Vorsteuerabzug ( 15 Abs. 1 Nr. 2 UStG). Der Ertrag der Einfuhrumsatzsteuer geht somit zu Lasten des Ertrags der Umsatzsteuer.

92 Statistisches Bundesamt: Fachserie 14/Reihe 8, Finanzen und Steuern, Umsatzsteuer, 2008, vom 31.03.2010, Zeitreihen 1, 1.1 Übersicht über steuerliche Merkmale.

93 Dieser Begriff wurde geprägt von Mittler, G.: Einführung von Vorsteuerbefreiungen als Mittel zur Umsatzsteuer-Betrugsbekämpfung, in: UR 2001, 385. 
Umsatzsteuer ist eine allgemeine Verbrauchsteuer, knüpft lediglich erhebungstechnisch an Leistungen des Unternehmers an. Die „Leistung an Verbraucher“ ist deshalb Tatbestandsmerkmal eines steuerbaren Umsatzes und setzt den Belastungsgrund gesetzestechnisch um. Das BStGB richtet die Umsatzsteuer deshalb von vornherein auf eine Belastung des Verbrauchers aus; grundsätzlich sind nur noch Leistungen an diesen steuerbar. Leistungen zwischen Unternehmern sind im Regelfall umsatzsteuerlich unerheblich. Die Umsatzsteuer aus einer Leistung zwischen Unternehmern entsteht nicht, ist nicht mehr in der Rechnung auszuweisen und nicht mehr vom Leistungsempfänger an den Leistenden zu bezahlen. Für den Verbraucher ergeben sich aus dieser Neuregelung keine Änderungen; er wird sie nicht bemerken.

Die Nichtsteuerbarkeit zwischenunternehmerischer Leistungen ist jedoch aus Gründen der Verlässlichkeit und Beweisbarkeit daran geknüpft, dass die Leistungspartner die Zahlung über Bankkonten abwickeln, die von der Finanzbehörde jederzeit eingesehen werden können (Gewährkonten) und dabei umsatzsteuerliche Identifikationsnummern verwenden, die ihnen von der Finanzbehörde zugeteilt werden. Die Verwendung der Gewährkonten bei Bezahlung durch Banküberweisung ist zwingend.

Das BStGB ist zwar als Gesetz für Deutschland formuliert, sein 3. Buch (Umsatzsteuer) ist jedoch ein Vorschlag an den europäischen Richtliniengeber. Deshalb soll die Nichtsteuerbarkeit zwischenunternehmerischer Leistungen in der gesamten EU gelten. So wird eine Regelung zur innergemeinschaftlichen Lieferung und zum innergemeinschaftlichen Erwerb überflüssig; alle zwischenunternehmerischen Leistungen werden gleich behandelt. Ausnahmsweise können Leistungen an Unternehmer dann steuerbar sein, wenn die Leistung nicht durch Banküberweisung bezahlt wird oder die Leistung vom Leistungsempfänger nicht ausschließlich unternehmerisch genutzt wird. Die Unternehmer erhalten in diesen Fällen den Vorsteuerabzug.

\section{Die öffentliche Hand}

Im geltenden Recht wird die öffentliche Hand zur Steuererhebung verpflichtet, wenn sie nach dem Maßstab des Körperschaftsteuerrechts als Betrieb gewerblicher Art gilt (§ 2 Abs. 3 Satz 1 UStG). Die Körperschaftsteuer und die Umsatzsteuer verfolgen jedoch unterschiedliche Zwecke: Die Körperschaftsteuer besteuert den Ertrag einer Körperschaft und belastet diesen Steuerschuldner in 
seinem Erfolg. ${ }^{94}$ Die Umsatzsteuer nimmt den Steuerpflichtigen nur erhebungstechnisch als Steuerschuldner in Anspruch, belastet aber den Verbraucher in seiner Kaufkraft. Die Verschonung der öffentlichen Hand bei der Umsatzsteuer entlastet den Verbraucher, der von der öffentlichen Hand Leistungen bezieht; diese Entlastung ist weder gewollt noch gerechtfertigt. Daher wird die Unterscheidung aufgegeben und die Besteuerung der öffentlichen Hand künftig einzig daran ausgerichtet, ob sie als Unternehmer am Markt Leistungen erbringt und Entgelte vereinnahmt oder im Rahmen öffentlicher Gewalt tätig wird. ${ }^{95}$

Bezieht die öffentliche Hand von einem Unternehmer eine Leistung, wird sie im geltenden Recht wie ein Verbraucher behandelt. Eine endgültige Belastung der öffentlichen Hand mit Umsatzsteuer ist jedoch systemwidrig. Die Besteuerung von Leistungen an die öffentliche Hand schafft kein Steueraufkommen, weil der Staat zugleich Steuerschuldner und Steuergläubiger ist. Die Umsatzsteuerzahlung einer Gebietskörperschaft, z.B. des Bundes, wirkt wie ein Finanzausgleich, weil ihr Ertrag zum Teil an den Bund zurückfließt, zum anderen Ländern und Gemeinden zusteht. Die Steuer stärkt nicht den Staatshaushalt, sondern belastet ihn. $^{96}$

Der Staat setzt beim Erwerb von Gütern Steuererträge ein, nicht privat erwirtschaftete Kaufkraft. Zudem nimmt der am Gütertausch beteiligte Staat eine Sonderstellung zwischen Unternehmern und Verbrauchern ein, wenn er nachfragt, um hoheitliche Aufgaben zu erfüllen. Der Staat kauft Waffen, um die öffentliche Sicherheit zu gewährleisten; baut Straßen, um den Bürgern Bewegungsmöglichkeiten zu erschließen; errichtet Hochschulinstitute, um Forschung und Lehre zu ermöglichen. Bei dieser Nachfrage wird der Staat eher als Unternehmer denn als Verbraucher tätig, weil er die Wirtschaftsgüter nicht für den staatseigenen Verbrauch, sondern zum Nutzen Dritter erwirbt. Allerdings ist er nicht als Unternehmer tätig, weil er im hoheitlichen Bereich keine Entgelte erzielt. Deswegen rechtfertigt die herkömmliche Begründung der Umsatzsteuer nicht, eine hoheitliche Staatstätigkeit zu belasten. Das bisherige Umsatzsteuersystem belastet jeden Nachfrager, mag er über Kaufkraft oder Steuerertragskraft verfügen, freiheitsberechtigt oder freiheitsverpflichtet sein, nach dem Belastungsgrund des Umsatzsteuerrechts als Steuerträger geeignet oder in seiner Hoheitsaufgabe ungeeignet sein. Dieses Fehlkonzept muss berichtigt werden: Der staatliche Hoheitsträger ist 
in allen seinen Untergliederungen grundsätzlich von vornherein nicht Steuerträger, sondern vermittelt nur bei staatlichen Leistungen die Steuerlast an den Nachfrager. Dieses Prinzip ist leicht durchführbar in einem Steuersystem, das zwischenunternehmerische Leistungen grundsätzlich freistellt, die Nichtsteuerbarkeit auf Rechnung und elektronische Identifizierung der Rechtsbeteiligten stützt.

\section{Steuerbefreiungen}

Die Steuerbefreiungen im Warenaustausch mit Drittstaaten bleiben erhalten. Die Ausfuhrlieferung ist steuerfrei, weil das Bestimmungsland die Einfuhr besteuert. Steuerbefreiungen bei der Einfuhr dienen zum Teil der Vereinfachung der Grenzabfertigung, folgen zum Teil internationalen Gepflogenheiten.

Im BStGB werden weitere Steuerbefreiungen gewährt, um das Leistungsangebot zu verbilligen. Hierzu gehören medizinische Leistungen, die Überlassung und Übertragung von Wohnraum, Versicherungen, die der persönlichen Zukunftssicherung dienen (z.B. Rentenversicherung, Lebensversicherung), und Umsätze, die einen gemeinnützigen Zweck zum Inhalt haben (z.B. Altenpflege, Jugendsport, Bildung). Finanzdienstleistungen bleiben ebenfalls in bisherigem Umfang steuerfrei.

Nach der Systematik des BStGB erwerben auch die Unternehmer, die steuerfreie Ausgangsleistungen erbringen, schon nichtsteuerbar. Der Vorsteuerabzug bleibt bei allen Steuerbefreiungen erhalten (,echte“ Steuerbefreiungen). Die von den Befreiungsvorschriften bezweckte Entlastung des Verbrauchers kommt somit voll zur Wirkung. Das Umsatzsteuergesetz und das Verwaltungsverfahren werden durch den Vorsteuerabzug erheblich vereinfacht: Er vermeidet eine komplizierte Vorsteueraufteilung ( $\$ 15$ Abs. 4 UStG) und Vorsteuerkorrektur ( $\$ 15 \mathrm{a}$ UStG), soweit ein Unternehmer sowohl steuerpflichtige als auch steuerfreie Umsätze ausführt. Die Steuerbefreiung des $\S 4 \mathrm{Nr} .28$ UStG wird überflüssig. Die Option ( 99 UStG) als Ersatzweg zu systematischen Ergebnissen - Vorsteuerentlastung in der Unternehmerkette - wird nicht mehr gebraucht, weil die Grundregel systemgerecht ausgestaltet ist.

Für Unternehmer mit Umsätzen bis 20.000 Euro wird eine Bagatellregelung beibehalten. Es besteht künftig jedoch kein Wahlrecht mehr, Umsatzsteuer dennoch zu erheben, abzuführen und Vorsteuern abzuziehen. 


\section{Ort der Leistung}

Die Umsatzsteuer belastet die vom Verbraucher am Markt eingesetzte Kaufkraft, die zu einem Leistungsaustausch führt. Nicht der tatsächliche Verbrauch begründet die Steuer, sondern der Erwerb des Verbrauchsguts. Der Belastungsgrund für die Umsatzsteuer verweist also von vornherein nur auf den Leistungsaustausch als marktoffenbaren Sachverhalt. ${ }^{97}$ Diesem Grundgedanken folgend ist Leistungsort der Ort, an dem die Leistung dem Empfänger erbracht wird.

Der Leistungsaustausch ist bei einer Lieferung von Gegenständen vollzogen, wenn dem Leistungsempfänger die Verfügungsmacht über den Gegenstand verschafft wird. Daher bestimmt die Grundregel als Leistungsort den Ort, an dem sich der gelieferte Gegenstand zu dem Zeitpunkt befindet, zu dem der leistende Unternehmer dem Leistungsempfänger die Verfügungsmacht über ihn verschafft (Verschaffungsort).

Der Leistungsaustausch ist bei einer Dienstleistung vollzogen, wenn sie dem Leistungsempfänger gegenüber erbracht wird. Daher ist Leistungsort grundsätzlich der Ort, an dem die Dienstleistung vom Leistungsempfänger empfangen wird (Empfangsort). Besondere Ortsregelungen für Dienstleistungen im Zusammenhang mit Grundstücken (Ort des Grundstücks), Arbeiten an beweglichen Gegenständen (Ort des Gegenstands), Dienstleistungen im Zusammenhang mit Veranstaltungen (Ort der Veranstaltung) konkretisieren diese Grundregel. Dienstleistungen, bei denen ein Empfangsort nur schwer zu ermitteln ist (z.B. auf elektronischem Weg erbrachte Dienstleistungen, Übertragung von Rundfunk und Fernsehen), werden am Sitz des Leistungsempfängers erbracht.

Ausnahmeregeln für Lieferungen von Gegenständen und Dienstleistungen an Bord von Beförderungsmitteln (Leistungsort ist der Abgangsort des Beförderungsmittels) und für die Beförderungsleistung (Leistungsort ist der Ort, an dem die Beförderung beginnt) dienen der Vereinfachung.

\section{Vorsteuerabzug}

Der Vorsteuerabzug hat aufgrund der weitgehenden Nichtsteuerbarkeit zwischenunternehmerischer Leistungen im BStGB nur noch eine sehr eingeschränkte Bedeutung. Er ist lediglich für Barumsätze bedeutsam. 
Gegenstände, die ursprünglich für den nichtunternehmerischen Bereich erworben wurden und später unternehmerisch genutzt werden, werden von der Umsatzsteuer entlastet; das BStGB erlaubt dem Unternehmer einen anteiligen Vorsteuerabzug auch bei der Einlage in das Unternehmen. Der nachträgliche Vorsteuerabzug bei der Einlage entspricht dem Sinn und Zweck des Vorsteuerabzugs, den Unternehmer von der Umsatzsteuer aus Eingangsleistungen zu entlasten, wenn und soweit sie für steuerbare Umsätze verwendet werden. Aus systematischen und praktischen Gründen werden Zeiträume benannt, bis zu deren Ablauf eine Entlastung von der Umsatzsteuer in Betracht kommt (,Gebrauchszeitraum“).

\section{Entstehung der Umsatzsteuer und des Vorsteuerabzugs}

Im geltenden Recht entsteht die Umsatzsteuer grundsätzlich mit Ablauf des Voranmeldungszeitraums, in dem eine Leistung erbracht wurde ( $\$ 13$ Abs. 1 Nr. 1 a) UStG). Spiegelbildlich ist der Abzug der geschuldeten Umsatzsteuer als Vorsteuer mit Ablauf des Voranmeldungszeitraums möglich, in dem eine Leistung empfangen wurde. Dies führt zu nicht gerechtfertigten Liquiditätsverschiebungen und Ausfallrisiken. Künftig hat der leistende Unternehmer die Umsatzsteuer dann abzuführen, wenn der Leistungsempfänger seine Kaufkraft eingesetzt hat. Er braucht als Helfer des Steuerträgers und der Verwaltung die Umsatzsteuer nicht mehr vorzufinanzieren. Der Leistungsempfänger erhält den Vorsteuerabzug nicht mehr, bevor er durch die gezahlte Umsatzsteuer tatsächlich belastet ist. Das vereinfacht auch erheblich das Abrechnungsverfahren, weil die Bemessungsgrundlagen zwischen Entstehen der Umsatzsteuer und deren Bezahlung nicht mehr korrigiert werden müssen. Es verringert die insolvenzbedingten Ausfälle, weil der Leistungsempfänger nicht mehr zwischen Vorsteuererstattung durch den Fiskus und Entgeltbezahlung an den Leistenden insolvent werden kann.

\section{Erbschaft- und Schenkungsteuer}

\section{Grundsätzlicher Reformbedarf}

Eigentum bleibt in der Generationenfolge nur in privater Hand, wenn die Garantie des Individualeigentums durch ein Erbrecht ergänzt wird. Würde das Privateigentum mit dem Tod des Eigentümers dem Staat zufallen, würden die Wirtschaftsgüter zunehmend in staatlicher Hand gesammelt; die Garantie des Privateigentums wäre strukturell ausgehöhlt. Deswegen gewährleistet Art. 14 Abs. 1 Satz 1 GG „Eigentum und Erbrecht“ in einem Atemzug. 
Eine ähnliche grundrechtszerstörende Wirkung würde eine Erbschaftsteuer erreichen, deren Höhe den Staat gleichsam zum Haupterben macht. Eine Steuer greift grundsätzlich dann zu, wenn der Steuerpflichtige unter Mitwirkung der Rechtsgemeinschaft leistungsfähiger geworden ist. ${ }^{98}$ Eine Besteuerung der Erbschaften und Schenkungen rechtfertigt sich, weil das Recht zu erben und zu schenken, dessen gerichtliche Durchsetzbarkeit, die von der Rechtsordnung garantierte Friedlichkeit des Erbanfalls und seiner späteren Nutzung dem Erben und Beschenkten eine neue Leistungsfähigkeit zuweist, die ihn in die Lage versetzt, finanziell zur Erhaltung des auch für ihn nützlichen Rechts-, Wirtschaft- und Gesellschaftssystems beizutragen.

Das Bundesverfassungsgericht hatte das geltende Erbschaft- und Schenkungsteuergesetz für verfassungswidrig erklärt, weil die Bewertung der Vermögensgüter dem Gleichheitssatz nicht entspreche. ${ }^{99}$ Der Gesetzgeber hat aufgrund der Entscheidung des Bundesverfassungsgerichts vom 07.11.2006 ${ }^{100}$ das Erbschaftund Schenkungssteuergesetz durch das Erbschaftsteuerreformgesetz vom 24.12.2008 ${ }^{101}$ grundlegend reformiert. Das Gesetz schafft allerdings nicht eine an Grundprinzipien des Steuerrechts ausgerichtete, systematische und allgemein verständliche Steuer, vielmehr eine kaum handhabbare Regelung, die viele Ausnahmen vorsieht und insbesondere Unternehmensvermögen für den Preis von Freistellungen und Steuerersparnissen langfristig rechtlich bindet. Der Reformbedarf wird dadurch aktueller.

\section{Rechtfertigung der Erbschaft- und Schenkungsteuer}

Die Erbschaft- und Schenkungsteuer wird wie kaum eine andere Steuerart grundlegend in Frage gestellt. Sie schaffe falsche Anreize gegen Vermögensbildung und lebenslange Erwerbsanstrengung, benachteilige Sparen und Vorsorge, sei sehr beratungs- und gestaltungsabhängig, zudem nur mit großem Aufwand und geringem Ertrag zu verwalten, die Steuersätze seien zu hoch. Gewichtige Stim-

98 Kirchhof, P.: Leistungsfähigkeit und Erwerbseinkommen, in: Festschrift für Joachim Lang, $2010,451$.

99 BVerfGE 93, 165; 117, 1 - Erbschaftsteuer.

100 BVerfGE 117, 1 - Erbschaftsteuer.

101 BGB1. I 2008, 3018. 
men fordern ihre Abschaffung, ${ }^{102}$ andere schlagen die Integration der Erbschaftsteuer in die Einkommensteuer vor. ${ }^{103}$

Die Erbschaft- und Schenkungsteuer rechtfertigt sich aus dem von der Rechtsordnung ermöglichten und gesicherten Zuwachs an Leistungsfähigkeit ${ }^{104}$ in seiner Ausprägung als Bereicherungsprinzip. ${ }^{105}$ Die Leistungsfähigkeit des Erben und Beschenkten ist durch den Reinvermögenszugang gestiegen. ${ }^{106}$ Der Vermögenszuwachs und dessen Nutzung stützen sich auf die vom Bürgerlichen Gesetzbuch gewährleistete Schenkungs- und Testierfreiheit, auf das gesetzliche Erbund Pflichtteilsrecht, auf den Rechtsschutz durch die staatlichen Gerichte, auf die staatliche Garantie von Sicherheit und Ordnung, von innerem und äußerem Frieden, die auch den Empfang größerer Erbschaften gefahrlos möglich macht. Auch der Erwerb und die Nutzung einzelner Wirtschaftsgüter - eines Grundstücks oder eines Gewerbebetriebs - setzt Funktionsgewährleistung und Sicherheit durch die öffentliche Hand voraus.

Das BStGB orientiert sich wieder strikt am Bereichungsprinzip. Die Steuer trifft nicht den Nachlass oder Schenker, sondern den Erben oder Beschenkten. Sie ist weiterhin in Form einer Erbanfallsteuer zu erheben. ${ }^{107}$ In der Hand der Erben war das Vermögen noch keiner Besteuerung ausgesetzt, eine Doppelbelastung liegt nicht vor. Die Erbschaftsteuer erfasst nicht - wie die Einkommensteuer - das Erwerbseinkommen, sondern die Vermögenszugänge durch Erbschaften und Schenkungen. ${ }^{108}$

102 Kruse, H.W.: Abschied von den Einheitswerten, in: BB 1996, 717 (719); Fischer, B.: Erbschaft- und Vermögenssteuer, Ein Beitrag zu den Steuerrechtfertigungslehren, in: StuW 1978, 345.

103 Lang, J.: Das verfassungsrechtliche Scheitern der Erbschafts- und Schenkungssteuer, in: StuW 2008, 189; Siegel, T.: Die Integration der Erbschaftsteuer in die Einkommensteuer auf der Grundlage des Realisationsprinzips, in: Festschrift für Jochen Sigloch, 2009, 445.

104 Zum Leistungsfähigkeitsprinzip vgl. BVerfGE 61, 319 (343 f.) - Ehegattensplitting; 82, 60 (86) steuerfreies Existenzminimum.

105 Der Gesetzgeber knüpft daher in $\S 10$ Abs. 1 Satz 1 ErbStG die Steuerpflicht an die Bereicherung.

106 Meincke, J.P.: Rechtfertigung der Erbschafts- und Schenkungssteuer, in: DStJG 22 (1999), 39 (40); Mellinghoff, R.: Das Verhältnis der Erbschaftsteuer zur Einkommen- und Körperschaftssteuer - Zur Vermeidung steuerlicher Mehrfachbelastungen, in: DStJG 22 (1999), 127 (135); Tipke, K.: Die Steuerrechtsordnung, a.a.O., 872; Hey, J.: BVerfG zur Erbschaftsteuer: Bewertungsgleichmaß und Gemeinwohlzwecke (Anmerkung zu BVerfG v. 07.11.2006 - 1 BvL 10/02), in: JZ 2007, 564 (573); Seer, R.: Die Erbschafts- und Schenkungssteuer im System der Besteuerung nach wirtschaftlicher Leistungsfähigkeit: Zugleich eine kritische Würdigung des Erbschaftsteuerreformgesetztes vom 24.12.2008, in: GmbHR 2009, 225 (226).

107 Nach der Reinvermögenszugangstheorie sind Erbschaft und Schenkung beim Erwerber als zugewendetes Einkommen zu besteuern, Lang, J.: in: Tipke, K./Lang, J., a.a.O., § 8, Rn. 38.

108 Tipke, K.: Die Steuerrechtsordnung, a.a.O., 874; Meincke, J.P., a.a.O., 39 (44). 
Denkbar wäre auch, sich von der Besteuerung von Erwerben von Todes wegen und Schenkungen zu lösen und alle Vermögensmehrungen (Reinvermögenszugänge) zu besteuern. Für eine solche allgemeine Bereicherungssteuer fehlt aber der rechtfertigende Grund, weil sie auch zufällige Vermögensmehrungen wie den Fund, ebenso die private Selbstbereicherung durch die Früchte im eigenen Garten oder die handwerkliche Leistung im eigenen Haus belasten würde, damit die Mitwirkung der Rechtsgemeinschaft am Entstehen dieser Bereicherung fehlt.

Das BStGB stellt die Erbschaft- und Schenkungsteuer als Belastung eines staatlich ermöglichten und gesicherten Vermögenszugangs neben die Einkommensteuer, die das Erwerbseinkommen belastet, und die Umsatzsteuer, die den Staat an der individuell eingesetzten Kaufkraft teilhaben lässt. So entsteht ein geschlossenes System der Besteuerung, ${ }^{109}$ das sich auf die staatlich gewährleistete und gestützte individuelle Leistungsfähigkeit ausrichtet.

\section{Verkehrswert als einziger Bewertungsmaßstab}

Bewertungsziel für alle Vermögensgegenstände ist der Verkehrswert. Nur er beschreibt die tatsächliche Bereicherung des Erwerbers als Ausdruck seiner hinzu gewonnenen Leistungsfähigkeit. In seinem Beschluss vom 07.11.2006 hat das BVerfG $^{110}$ auf eine Vorlage des $\mathrm{BFH}^{111}$ die Erhebung der Erbschaft- und Schenkungsteuer nach $\S 19$ Abs. 1 ErbStG für mit dem Grundgesetz unvereinbar erklärt, weil einheitliche Steuersätze auf jeden Vermögenserwerb angewendet werden, obwohl die Bemessungsgrundlagen nach Vorschriften ermittelt würden, die nicht den Anforderungen des Gleichheitssatzes des Art. 3 Abs. 1 GG genügten. Das BVerfG stellte fest, die Bewertung jedes anfallenden Vermögens habe sich am gemeinen Wert als Bewertungsziel zu orientieren.

Der Verkehrswert entspricht als Richtschnur der Wertfeststellung dem Bereicherungsprinzip: In der Form der Erbanfallsteuer knüpft die Erbschaftsteuer an den Übergang von Vermögenssubstanz vom Erblasser auf den Erben und die diesem dadurch zufallende Bereicherung an. Die Steuer muss sich daher nach dem Wert der übergehenden Vermögenssubstanz bemessen. Die Erbschaft ist das wert, was sich aus ihrer Substanz bei Veräußerung erlösen lässt. ${ }^{112}$ Für die Schenkungsteu- 
er gilt das Gesagte entsprechend. Jedes zugewandte Vermögen, gleich ob inländisches oder ausländisches Wirtschaftsgut, muss mit dem Verkehrswert erfasst werden. Die Gleichbehandlung jeglichen Vermögens beseitigt auch europarechtliche Bedenken, denen die heutige Unterscheidung von Inlands- und Auslandsvermögen begegnet.

\section{Praktikable Bewertungsverfahren}

Die Vorschriften über die Bewertung für Zwecke der Erbschaft- und Schenkungsteuer werden neu gefasst und in das Erbschaftsteuergesetz eingefügt. Das Bewertungsrecht wird dadurch wieder übersichtlich und einfacher zu handhaben.

Bemessungsgrundlage des BStGB ist die Summe der Steuerwerte aller erworbenen Vermögensgüter abzüglich der die Bereicherung vermindernden Steuerwerte. Steuerwert ist der Wert, mit dem ein Vermögensgut in die Bemessungsgrundlage eingeht. Wie dieser Wert ermittelt wird, hängt von der Art des Vermögensgutes ab: Bei Geldvermögen gilt der Nominalwert, bei anderen Vermögensarten, wie Grundvermögen oder Unternehmensvermögen, stellt ein besonderes Bewertungsverfahren den Wert fest. Die Steuerwerte einiger Vermögensgüter werden pauschaliert. Das ist verfassungsrechtlich unbedenklich. Der Gesetzgeber kann zur Vereinfachung steuerlicher Massenverfahren eine Gleichheit im Typus herstellen, ${ }^{113}$ die den Sachverhalt vergröbert erfasst oder steuerliche Belastungstatbestände pauschalierend regelt. ${ }^{114}$

Der Steuerwert ist eine einheitliche Bewertungskategorie des Erbschaft- und Schenkungsteuerrechts im BStGB. Er nimmt den Verkehrswert eines Vermögensgutes zum Maßstab. Die typisierten Bewertungsverfahren, die zum Steuerwert führen, orientieren sich am Begriff des Verkehrswertes als dem Veräußerungspreis im gewöhnlichen Geschäftsverkehr, finden in diesem ihren Rahmen. Der für alle Vermögensgüter einheitliche Wertbegriff des Steuerwertes sucht sich stets dem einheitlichen Maßstab des Verkehrswertes anzunähern, auch wenn die Güter nicht getauscht, nicht in der Rationalität des gegenseitigen Vertrages der vereinbarten Angemessenheit - von den Beteiligten bewertet werden. Das BStGB erfüllt damit die zentrale Forderung des BVerfG nach einer gleichmäßigen Bewertung aller Vermögensarten als Voraussetzung einer verfassungsgemäßen Erbschaft- und Schenkungsteuer. 


\section{Bewertung erst mit tatsächlicher Verfügungsbefugnis}

Im BStGB gilt das Stichtagsprinzip. Die übergegangenen Vermögensgüter werden zu dem Zeitpunkt bewertet, an dem die Steuer entsteht. Derselbe Stichtag bestimmt die persönliche Steuerpflicht und den Beginn der Verjährung. Die Wertermittlung auf den Stichtag der Steuerentstehung nimmt allerdings nachträgliche Wertermittlungen nicht auf. Die Bemessungsgrundlage und die Bereicherung, die dem Erwerber tatsächlich zufließt, weichen von einander ab, wenn erworbene Vermögensgüter an Wert verlieren. Im geltenden Erbschaft- und Schenkungsteuerrecht führt dieser Widerspruch zwischen Bereicherungsprinzip und Stichtagsprinzip immer wieder zu Streitfällen. Zudem kann mancher Erwerber aufgrund des Verhaltens eines Dritten oder eines Streits um seine Berechtigung erst lange nach dem Stichtag über das ihm zugewandte Vermögen verfügen. Gleiches gilt für Erwerber bestimmter Vermögensgüter, insbesondere börsennotierter Wertpapiere oder Edelmetalle, die in ihrem Wert stark schwanken.

Das BStGB betont das Bereicherungsprinzip. Es genießt gegenüber dem Stichtagsprinzip Vorrang. Deshalb wird die Steuerentstehung auf den Zeitpunkt verschoben, in dem der Erwerber rechtlich über das ihm zugewandte Vermögen verfügen kann. Das betrifft vor allem Fälle, in denen die Verfügungsmöglichkeit des Erwerbers vom Verhalten eines Dritten, meist des Erben, abhängt. Erst wenn der Erwerber tatsächlich über ein Vermögensgut verfügen kann, ist er bereichert, damit leistungsfähiger und besteuerungswürdig. Ein Vermögensgut geht deshalb nur mit dem Wert in die Bemessungsgrundlage der Steuer ein, den es in diesem Zeitpunkt hatte.

Die Steuer ist entsprechend zu mindern, wenn ein Erwerber am Bewertungsstichtag tatsächlich noch nicht in der Lage ist, über ein Vermögensgut zu verfügen, und es bis dahin erheblich an Wert verliert. Auch in Fällen, in denen rechtlich die Verfügungsmacht schon auf den Erwerber übergegangen ist, hat also das Bereicherungsprinzip Priorität vor dem Stichtagsprinzip. Besteuert wird die Bereicherung, die tatsächlich im Vermögen des Erwerbers greifbar ist. Der Erwerber hat einen Anspruch darauf, nach seinem tatsächlichen, nicht nur vermuteten $\mathrm{Zu}$ wachs an Leistungsfähigkeit besteuert zu werden. 


\section{Nur noch Erbschaften und Schenkungen als abstrakte Erwerbs- tatbestände}

Das BStGB regelt die steuerlichen Belastungsgründe eigenständig und löst sich von den Verweisen auf das BGB. Die Erwerbstatbestände werden eigenständig gefasst und abstrakt formuliert. Das BStGB erfasst im Tatbestand des Erwerbs von Todes wegen jeden durch den Tod des Erblassers verursachten Vermögensanfall, der den Erwerber bereichert. Dieser Tatbestand hebt den Belastungsgrund, die Bereicherung, hervor. Die Erwerbstatbestände folgen dem Grund der Steuerlast, dem unentgeltlichen Erwerb zusätzlicher Leistungsfähigkeit. Der Erwerb wird unabhängig von Besonderheiten des Einzelfalls und steuerplanender Gestaltung besteuert.

Das Erbschaft- und Schenkungsteuerrecht besteuert die Bereicherung, die einem Erwerber von Todes wegen oder einem Beschenkten zugewendet worden ist. Die rechtliche Form der Zuwendung ist unerheblich. Auch kommt es nicht darauf an, ob sich das Vermögen im In- oder Ausland befindet. Durch die abstrakte Formulierung der Erwerbstatbestände erübrigt sich der Vergleich ausländischer Zivilrechtsformen mit den deutschen. ${ }^{115}$ Die Erwerbe aufgrund ausländischer Zivilrechtsordnungen sind immer steuerbar, wenn sie die Erwerbstatbestände des ErbStGB erfüllen.

Die Ersatzerbschaftsteuer soll entfallen. Bisher unterliegt in Zeitabständen von jeweils 30 Jahren das Vermögen einer Stiftung oder eines Vereins, sofern diese wesentlich im Interesse einer Familie oder bestimmter Familien errichtet oder auf Bindung von Vermögen für diesen Personenkreis gerichtet sind, der Erbschaftsteuer. Die Ersatzerbschaftsteuer bei Familienstiftungen widerspricht dem Grundgedanken der Erbanfallsteuer. Die Ersatzerbschaftssteuer besteuert nicht die Bereicherung des Bezugsberechtigten, sondern die Stiftung, die um die Vermögensausschüttungen an die Destinatäre entreichert ist. Außerdem werden Familienstiftungen höher besteuert als die übrigen Stiftungen und auch ausländischen Familienstiftungen. Während Familienstiftungen oder -vereine der Ersatzerbschaftsteuer unterliegen, gilt dies für alle anderen Stiftungen und Vereine nicht. Diese Ungleichbehandlung ist nicht zu rechtfertigen.

Das BStGB verzichtet auch auf den Erwerbstatbestand der Zweckzuwendung. Als Zweckzuwendungen besteuert das geltende Recht Zuwendungen von Todes

115 Siehe dazu für das geltende Recht Klein, M.: Die Typologie des Erbschaftssteuergesetzes bei Auslandserwerben - zugleich ein Beitrag zur wirtschaftlichen Betrachtungsweise im Erbschaftssteuerrecht, in: FR 2001, 118. 
wegen oder Zuwendungen unter Lebenden, die mit der Auflage verbunden sind, zugunsten eines bestimmten Zwecks verwendet zu werden, soweit hierdurch die Bereicherung des Erwerbers gemindert wird. Die Zweckzuwendung unterscheidet sich von der Schenkung dadurch, dass bei der Zweckzuwendung ein Vermögen zugewandt wird, das nicht für eigene, sondern für fremde Zwecke oder einen unbestimmten Personenkreis zu verwenden ist. Besteuert wird also nicht die Bereicherung einer Person, sondern die Tatsache, dass einer Person etwas zugewandt wurde, das zumindest mit einem Teil einen bestimmten Zweck erfüllen muss. Bei der Zweckzuwendung ist der Empfänger nicht bereichert. Besteuert wird aber nicht der letztlich Bereicherte, sondern der mit der Auflage Belastete. Dieses Ergebnis ist mit dem Bereicherungsprinzip und dem Postulat der Besteuerung nach der Leistungsfähigkeit nicht zu vereinbaren.

\section{Steuerfreie Zuwendungen unter Ehegatten und eingetragenen Lebens- partnern}

Im geltenden Steuerrecht sind Zuwendungen unter Ehegatten grundsätzlich steuerpflichtig. Dabei wird übersehen, dass die Ehe neben einer Lebens- auch eine Erwerbsgemeinschaft darstellt, in der beide Ehepartner durch ihre Leistungen gemeinsam ihr Vermögen mehren. Das Ehegut bleibt beim Tod eines Ehegatten in der Ehe. Die Erbschaftsteuer besteuert regelmäßig die Bereicherung bei einem Generationenwechsel. Wenn einer der beiden Ehepartner verstirbt, tritt ein solcher Generationenwechsel beim überlebenden Ehegatten nicht ein. Deshalb sind Zuwendungen unter Ehegatten von der Besteuerung auszunehmen. Die Steuerfreiheit von Ehegattenzuwendungen vermeidet die im bestehenden Recht oft sehr schwierigen Abgrenzungsfragen zwischen Steuerrecht und ehelichem Güterrecht, vor allem bei der Behandlung des Zugewinnausgleichs.

Aus Gründen der Gleichbehandlung soll die Steuerfreiheit von Ehegattenzuwendungen auch für Zuwendungen unter eingetragenen Lebenspartnern gelten. Da das Gesetz über die eingetragene Lebenspartnerschaft $\left(\mathrm{LPartG}^{116}\right)$ die Rechte und Pflichten der Lebenspartner untereinander zivilrechtlich denen der Ehegatten angleicht, werden ihnen auch steuerlich die gleichen Rechte wie Ehepartnern eingeräumt.

116 Gesetz über die Eingetragene Lebenspartnerschaft (LPartG) vom 16. 2. 2001 (BGB1. I 2001, 266), zuletzt geändert durch Art. 7 des Gesetzes zur Änderung des Zugewinnausgleichs- und Vormundschaftsrechts vom 6. 7. 2009 (BGB1. I 2009, 1696). 


\section{Beschränkung der Steuerbefreiungen}

Grundsätzlich ist im BStGB jede Bereicherung durch Erwerb von Todes wegen und durch Schenkung steuerbar und auch steuerpflichtig. Steuerbefreiungen bedürfen als Ausnahmen hiervon einer besonderen Rechtfertigung. Der Katalog der Steuerbefreiungen im BStGB ist auf solche beschränkt, die aus dogmatischen, systematischen oder praktischen Gründen notwendig sind. Das sind insbesondere Zuwendungen an gemeinnützige, mildtätige und kirchliche Organisationen, an Gebietskörperschaften und Zuwendungen von Hausrat und üblichen Gelegenheitsgeschenken.

Bei einer Bereicherungssteuer wird der Schwerpunkt der Entlastung von den gegenständlichen Befreiungen hin zu den persönlichen Freibeträgen verlagert. Die Frage, ob der jeweilige Erwerber mit einer Steuer belastet werden soll, darf nicht schon in der Bemessungsgrundlage beantwortet werden, sondern betrifft die Frage, ob dem Erwerber aus persönlichen Gründen die Steuerzahlungslast zugemutet werden kann.

\section{Persönliche Freibeträge}

Das BStGB hat zum Ziel, die erhöhte Leistungsfähigkeit, die tatsächliche Bereicherung folgerichtig zu besteuern. Die Garantie des Erbrechts in Art. 14 Abs. 1 Satz 1 GG gebietet aber, einen gewissen Teil der Zuwendung dem Erwerber ungeschmälert zufließen zu lassen. Die Erbschaftsteuer darf die Funktion des Erbrechts als Rechtsinstitut und Individualgrundrecht nicht zunichte oder wertlos machen. ${ }^{117}$ Die Testierfreiheit des Erblassers und das Verwandtenerbrecht dürfen durch die Steuerbelastung nicht ausgehöhlt werden. ${ }^{118}$ Dem Erwerber muss deshalb die Möglichkeit eröffnet werden, persönliche Freibeträge von der Bemessungsgrundlage abzuziehen. Schließlich sprechen auch Gründe der Verwaltungsökonomie für die Einführung von Freibeträgen, ${ }^{119}$ um zu verhindern, dass auch Erbschaften oder Schenkungen in geringer Höhe eine Veranlagung veranlassen.

Allerdings können auch die Freibeträge vereinfacht werden. Zwei statt der bisher sieben verschiedenen persönlichen Freibeträge ${ }^{120}$ sind ausreichend. Zum einen erhalten Kinder einen Freibetrag von 400.000 Euro. Dieser Freibetrag für Kinder 
- ergänzt durch die Befreiung des Hausrats bis zu einer Höhe von 20.000 Euro verwirklicht das Familienprinzip, das aus Art. 6 Abs. 1 GG folgt: Das Familiengut ist etwa im Wert des persönlichen Gebrauchsvermögens von der Besteuerung auszunehmen. ${ }^{121}$ Zum anderen bleiben Zuwendungen an alle übrigen Bedachten bis zu einem Betrag von 50.000 Euro als Bagatellfälle von der Besteuerung frei. Damit wird vermieden, dass jeder, auch der kleinere Vermögensübergang zu einem Besteuerungsfall wird, bewertet und veranlagt werden muss.

\section{Niedriger und einheitlicher Steuersatz}

Die Bemessungsgrundlage des BStGB verbreitert sich, weil realitätsgerecht bewertet wird und die Steuerbefreiungen im Wesentlichen entfallen. Das ermöglicht einen im Vergleich zum geltenden Recht beachtlich verringerten einheitlichen Steuersatz.

Gleichzeitig werden die verschiedenen Steuerklassen überflüssig. Das Ehegut und das Familiengut werden nicht mehr durch ein gestuftes System von unterschiedlichen Steuerklassen geschützt, sondern durch die Steuerbefreiung von Erwerben unter Ehegatten und die erhöhten Freibeträge für den Erwerb von Kindern des Erblassers oder Schenkers.

\section{Liquiditätsprobleme bei nicht verfügbaren Vermögen}

Alle Bereicherungen werden ohne Unterscheidung nach verschiedenen Vermögensarten gleichermaßen besteuert. Für den Übergang von Vermögen können folgerichtig keine Erleichterungen mehr eingeräumt werden. Solche Differenzierungen würden ohnehin die Erbschaftsteuerlast von Erwerbern entlasteten Vermögens (Betriebsvermögen) auf Erwerber regelbelasteten Vermögens (z.B. Geldvermögen) verschieben. Unternehmerisch gebundenes Vermögen muss nach den Vorgaben des BVerfG wie alle anderen Vermögen mit dem Verkehrswert bewertet werden. ${ }^{122}$ Tatsächliche Unterschiede, z.B. des betrieblichen oder landund forstwirtschaftlichen Vermögens, betreffen die Bewertung.

Nach der Rechtsprechung des BVerfG bleibt es dem Gesetzgeber jedoch unbenommen, durch Verschonungsregeln den Erwerb bestimmter Vermögensgegenstände zu entlasten, wenn dafür ausreichende Gemeinwohlgründe vorliegen. ${ }^{123}$ 
Schon in seinem Beschluss vom 22.06.1995 wies das Gericht darauf hin, dass die wirtschaftliche Existenz bestimmter Betriebe durch die finanzielle Belastung mit Erbschaft- und Schenkungsteuer gefährdet sein könne. ${ }^{124}$ Derartige Betriebe seien durch das Wirtschaftsverwaltungsrecht und das Arbeitsrecht gesteigert gemeinwohlgebunden und gemeinwohlverpflichtet. Betrieblich gebundenes Vermögen sei in seiner Verfügbarkeit beschränkter als anderes Vermögen. Infolgedessen entspräche die finanzielle Leistungsfähigkeit des Erben nicht in voller Höhe seinem Vermögenszuwachs. ${ }^{125}$

Diese Überlegungen fließen im Verkehrswert schon in die Bewertung ein, da der Markt alle Tatsachen berücksichtigt, die für die Preisbildung von Bedeutung sind, also auch die Sozialpflichtigkeit eines Unternehmens. ${ }^{126}$ Dennoch ist die verminderte Verfügbarkeit betrieblich gebundenen Vermögens bei der Besteuerung zu beachten. Die Erbschaft- und Schenkungsteuer mutet einem Erwerber grundsätzlich zu, zumindest Teile des Erworbenen zu veräußern, um die Steuer begleichen zu können. Teile eines betrieblichen Vermögens lassen sich jedoch oft nur schwer und unter finanziellen Verlusten zu Geld machen. Gerade die betriebliche Gebundenheit verschiedener Wirtschaftsgüter macht ihren Wert aus. Deshalb wird betriebliches Vermögen als Gesamtheit bewertet und nicht als Summe einzelner Werte angesehen.

Das BStGB lockert den Steuerzugriff deshalb im Rahmen des Erhebungsverfahrens. Die Steuer kann auf höchstens zehn Jahre gestundet werden. Die Stundung wird auf Antrag gewährt. Der Steuerpflichtige hat einen Anspruch auf die Stundung. Die gestundete Steuer ist nicht zu verzinsen.

\section{VIII.Verbrauchsteuer}

\section{Das Konzept der Verbrauchsteuern}

Im geltenden Recht bestehen neben der Umsatzsteuer als allgemeiner Verbrauchsteuer zahlreiche besondere Verbrauchsteuergesetze. Sondergesetze besteuern Energie und Strom, deren Ertrag sich auf ca. 46 Mrd. Euro beläuft, Tabak mit einem Aufkommen von ca. 14 Mrd. Euro, Branntwein, Kaffee, Bier,

124 BVerfGE 93, 165.

125 BVerfGE 93, 165 (175 f.).

126 Bareis, P.: Probleme verfassungsrechtlicher Vorgaben und ihrer Umsetzung am Beispiel der Vermögen- und Erbschaftsteuer, in: DB 1996, 1153 (1157); Bach, S./Broekelschen, W./Maiterth, R.: Gleichmäßige erbschaftssteuerliche Behandlung von Grund- und Betriebsvermögen, in: DStR 2006, 1961 (1966). 
Schaumwein, Zwischenerzeugnissen und „Alkopops“ mit einem Aufkommen von insgesamt ca. 4 Mrd. Euro. ${ }^{127}$ Der Ertrag steht - mit Ausnahme der Biersteuer - dem Bund, ein geringer Anteil des Aufkommens aus der Energiesteuer den Ländern zu. Gegenständlicher Anknüpfungspunkt einer besonderen Verbrauchsteuer ist ein Produkt. ${ }^{128}$ Die Steuer entsteht mit deren Verbringen in den steuerlich freien Verkehr, also in der Regel der Entnahme aus einem Steuerlager (Art. 6 Abs. 1 VerbrStSystRL). An diesem bewährten System hält der vorgelegte Reformvorschlag fest.

Die Umsatzsteuer erfasst die am Markt eingesetzte Kaufkraft als Vorgriff auf den folgenden, aber vom Markt nicht mehr beobachtbaren Verbrauch des erworbenen Gutes. Sie besteuert die Einkommensverwendung des Verbrauchers, weil diese Leistungsfähigkeit vermuten lässt. Sie wird zwar nicht - wie bei der Besteuerung des Einkommenszuwachses - individuell ermittelt, aber typisierend unterstellt.

Die besonderen Verbrauchsteuern belasten ebenso den Verbrauch bestimmter Güter. ${ }^{129}$ Der EuGH definiert die Verbrauchsteuer als eine Steuer, die nur auf ganz bestimmte Gegenstände und nur auf einer Stufe des Herstellens oder Transports ohne die Gewährung eines Vorsteuerabzugs erhoben wird. ${ }^{130}$ Die bestimmten Gegenstände zeichnen sich dadurch aus, dass sie zur Deckung ständigen Bedarfs kurzfristig verbraucht werden. ${ }^{131}$ Bezeichnend für eine Verbrauchsteuer ist, dass ein spezielles Gut mit dem Herstellen oder Verbringen ins Inland grundsätzlich in einen steuergebundenen Raum tritt und mit Verbringen in den steuerlich freien Verkehr - unabhängig von einem Rechtsakt und dem Einsatz von Kaufkraft - besteuert wird. ${ }^{132}$ Das heutige System krankt jedoch daran, dass die Auswahl der Güter lediglich historisch begründet werden kann. ${ }^{133}$

127 Die Zahlen beziehen sich auf das Jahr 2006, vgl. BMF: Finanzbericht 2008, 288.

128 Vgl. Jatzke, H.: Das System des deutschen Verbrauchsteuerrechts, 1997, 46.

129 Vgl. BVerfGE 98, 123.

130 Vgl. EuGHE 1989, 2671 (2707).

131 Vgl. BVerfGE 98, 106, 123 f. - Kommunale Verpackungssteuer.

132 Vgl. BFHE 57, 473 (489); 141, 369 (373).

133 Vgl. Tipke, K.: Die Steuerrechtsordnung, a.a.O., 1037 ff.; Lang, J., in: Tipke, K./Lang, J., a.a.O., § 8, Rn. 106. 


\section{Auswahl der zu besteuernden Güter}

Der Reformvorschlag wählt die zu besteuernden Güter nach den durch ihre Verwendung entstehenden Kosten aus. Ist ein umweltschädliches oder (dritt-)schädigendes Verhalten nicht hinnehmbar, ist es grundsätzlich zu unterbinden. Ist ein solches Verhalten noch hinnehmbar, kann im Wege der Verteuerung des Produkts dem Marktteilnehmer die Entscheidung für oder gegen dieses - noch tolerierbare - Verhalten überlassen, aber ein finanzieller Ausgleich hierfür abverlangt werden.

Die Energiesteuer ist eine Sonderlast für den Verbrauch eines Gutes, das im Gemeinwohlinteresse zu erhaltende Ressourcen der „Umwelt“ mindert. Dieser Ressourcenverbrauch geht nicht in den Preis eines Produkts ein, weil durch die Inanspruchnahme der Umwelt als Allgemeingut für die Marktteilnehmer keine Kosten entstehen. Dieses Gut hat jedoch seinen Preis. Die Besteuerung der Energie und des Stroms lässt sich daher damit rechtfertigen, dass derjenige, der diese Güter verbraucht, eine erhöhte Gemeinwohlverantwortung trägt. Hierfür soll eine Steuer entrichtet werden. Die Tabak- und Alkoholsteuer fordern einen Ausgleich für die Kosten, die der Allgemeinheit typischerweise durch den Genuss von Tabak und Alkohol entstehen können.

Die vorgegebenen Güter werden in den geltenden EU-Verordnungen und entsprechend in den deutschen Gesetzen überwiegend nicht definiert, sondern durch Bezugnahme auf die Kombinierte Nomenklatur des Zolls ${ }^{134}$ benannt. Der Reformvorschlag wählt hier einen anderen Weg. Er schafft für die Anwender Klarheit. Er benennt die Güter, die mit einer Verbrauchsteuer belastet werden und verweist nicht auf andere Gesetzeswerke.

\section{Europarechtliche Vorgaben}

In der EU existiert die Verbrauchsteuer-Systemrichtlinie, ${ }^{135}$ die durch Strukturund Steuersatzrichtlinien ergänzt wird, die Verbrauchsteuern in Europa harmonisiert hat und für die Mitgliedstaaten die Pflicht begründet, Energie, Alkohol,

134 Kombinierte Nomenklatur ab dem 1.1.2011: Verordnung (EU) Nr. 861/2010 der Kommission vom 5. Oktober 2010 zur Änderung von Anhang I der Verordnung (EWG) Nr. 2658/87 des Rates über die zolltarifliche und statistische Nomenklatur sowie den Gemeinsamen Zolltarif.

135 Richtlinie Nr. 92/12 (EWG) des Rates über das allgemeine System, den Besitz, die Beförderung und die Kontrolle verbrauchsteuerpflichtiger Waren vom 25.2.1992, AB1. EG Nr. L 76, 1, ber. AB1. EG 1995 Nr. L 17 20, folgend: VerbrStSystRL. 
alkoholische Getränke und Tabak der Verbrauchsbesteuerung zu unterwerfen. ${ }^{136}$ Für alkoholische Getränke gilt die Ausnahme, dass die Mitgliedstaaten einen Null-Steuersatz für stillen Wein und Schaumwein vorsehen dürfen. ${ }^{137}$ Darüber hinaus gibt es eine Einfuhr-Verbrauchsteuerbefreiungs-VO, ${ }^{138}$ EinreiseFreimengen- $\mathrm{VO}^{139}$ und eine Kleinsendungs-Einfuhrmengen-VO. ${ }^{140}$

Diese europarechtlichen Vorgaben entziehen den Mitgliedstaaten die Befugnis, die Besteuerung der genannten Produkte in eigener Verantwortung zu regeln. Änderungen der bestehenden Regelungen bedürften der Einstimmigkeit. ${ }^{141}$

Im Gesamtkonzept eines Bundessteuergesetzbuches wird zunächst ein nationales Verbrauchsteuergesetzbuch vorgestellt. Es ist jedoch auch als Reformvorschlag an den europäischen Gesetzgeber gedacht. Das Verbrauchsteuergesetzbuch ist so verfasst, dass es auch als europäischer Rechtsakt erlassen werden könnte.

Der Reformvorschlag kann auf europäischer Ebene am besten durch eine Richtlinie umgesetzt werden. Art. 113 AEUV enthält für die Handlungsform keine Vorgaben; ${ }^{142}$ er überlässt die Wahl der Handlungsform dem zuständigen Organ. ${ }^{143}$ Die Richtlinie bietet sich an, weil sie das Ziel verbindlich regelt, jedoch die Mittel den Mitgliedstaaten überlässt. Sie achtet den Entscheidungsraum und die Rechtsstrukturen der Mitgliedstaaten.

Der Reformvorschlag verfolgt das Ziel, zur Verwirklichung des Binnenmarktgedankens das VerbrStGB als unmittelbar geltendes Recht in der gesamten Europäischen Union zur Anwendung zu bringen.

136 Vgl. Art. 3 Abs. 1 VerbrStSystRL, Art. 1 der Richtlinie (EG) 2003/96 des Rates zur Restrukturierung der gemeinschaftlichen Rahmenvorschriften zur Besteuerung von Energieerzeugnissen und elektrischem Strom vom 27.10.2003 (AB1. EU Nr. L 283, 51, folgend: EnergieStRL.

137 Vgl. Art. 5 der Richtlinie (EWG) Nr. 92/84 des Rates über die Annäherung der Verbrauchsteuersätze auf Alkohol und alkoholische Getränke vom 19.10.1992, ABl. EG Nr. L 316, 29, folgend. AlkStSatzRL.

138 Einfuhr-Verbrauchsteuerbefreiungsverordnung (EVerbrStBV), BGB1. I 1999, 1414.

139 Verordnung über die Einfuhrabgabenfreiheit von Waren im persönlichen Gepäck von Reisenden, Einreise-Freimengen-Verordnung (EF-VO), BGB1. I 2008, 2235.

140 Verordnung über die Einfuhrabgabenfreiheit von Waren in Sendungen von Privatpersonen an Privatpersonen, Kleinsendungs-Einfuhrfreimengen-Verordnung (KF-VO), BGB1. I 1979, 73.

$141 \mathrm{Vgl}$. Jatzke, H., a.a.O., 28.

142 Vgl. Voß, R., in: Grabitz, E./Hilf, M. (Hg.): Kommentar zum EG-Vertrag, 2010, 42. Auflage, Art. 93 Rn. 12; Jatzke, H., a.a..O., 28.

143 Vgl. Nettesheim, M., in: Grabitz, E./Hilf, M. (Hg.), a.a.O. Art. 249 Rn. 78, der jedoch im Rahmen des Art. 93 EGV lediglich die Richtlinie als die geeignete Handlungsform betrachtet. 
Reformgedanken im Steuerrecht hoffen auf die Reformfähigkeit und Reformbereitschaft der deutschen Demokratie, deren Ergebnisse dann europäische Initiativen zur Folge haben mögen. Sie wenden sich an die Bürger und Steuerpflichtigen, die das Steuerrecht wieder zu einer Quelle allgemeinen Rechtsbewusstseins machen wollen. Auch im Steuerrecht muss der ehrbare Kaufmann, der redliche Bürger wieder verlässlich wissen, was sich gehört. 